\title{
Perturbation Solution for Radiating Viscoelastic Fluid Flow and Heat Transfer with Convective Boundary Condition in Nonuniform Channel with Hall Current and Chemical Reaction
}

\author{
B. J. Gireesha and B. Mahanthesh \\ Department of Studies and Research in Mathematics, Kuvempu University, Shankaraghatta, Shimoga, Karnataka, 577 451, India
}

Correspondence should be addressed to B. J. Gireesha; bjgireesu@rediffmail.com

Received 24 May 2013; Accepted 4 July 2013

Academic Editors: G. L. Aranovich, C. D. Daub, and A. Ghoufi

Copyright (C) 2013 B. J. Gireesha and B. Mahanthesh. This is an open access article distributed under the Creative Commons Attribution License, which permits unrestricted use, distribution, and reproduction in any medium, provided the original work is properly cited.

\begin{abstract}
A mathematical analysis has been performed for heat and mass transfer of a time-dependent MHD flow of an electrically conducting viscoelastic fluid in nonuniform vertical channel with convective boundary condition. The fluid flow is considered between a vertical long wavy wall and a parallel flat wall saturated with the porous medium. The effects of thermal radiation, heat absorption, chemical reaction, and Hall current are taken into account. The prevailing nonlinear partial differential equations are derived by considering Boussinesq approximation, and the same equations are solved analytically using perturbation technique. Further the expressions for skin friction, Nusselt number, and Sherwood number are presented. The effects of various pertinent parameters on different flow fields are analyzed graphically and tabularly. It is found that effects of Hall parameter and Biot number are unfavorable on velocity profiles, but this trend is reverse for the effect of thermal and solutal Grashof numbers. The expressions of different flow fields satisfy the imposed boundary conditions, which is shown in all graphs; this implies accuracy of the solution.
\end{abstract}

\section{Introduction}

The study of viscoelastic fluid has become important in the last few years. Qualitative analysis of these studies has significant bearing on several industrial applications such as polymer sheet extrusion from a dye and drawing of plastic firms. When manufacturing processes at high temperature need cooling, the flow may need viscoelastic fluid to produce a good effect or reduce the temperature. On the other hand, the flow and heat transfer of a viscoelastic fluid between parallel plates have significant role in many engineering fields such as petroleum production, chemical catalytic reactors, and solar power collectors. Boundary layer treatment for an idealized viscoelastic fluid was introduced by Beard and Walters [1]. There has been a continued interest in the investigation of natural convection heat transfer of non-Newtonian fluid, which exhibits the viscoelasticity. Recently, Rajagopal and $\mathrm{Na}$ [2] have studied the heat transfer analysis in the forced convection flow of a visco-elastic fluid by considering the Walters model. The problem of MHD flow and heat transfer has wide range of applications in emerging fields due to an electro-magnetic field are relevant to many practical applications in geophysical and astrophysical situations, the metallurgy industry, and cooling of continuous strips and filaments drawn through a quiescent fluid. Sarpkaya [3] was the first who had studied the MHD flows in non-Newtonian fluids. Thereafter, several authors [4-10] have shown their interest in MHD flows under different physical conditions.

Convective flow with simultaneous heat and mass transfer under the influence of a magnetic field and chemical reaction has attracted considerable attention of many researchers because of its applications in various branches of science and technology. On the other hand, radiative heat transfer has many applications in nuclear power plants, in gas turbines, 
and in the various propulsion devices for space vehicles, missiles, and aircrafts. Motivated by these applications very recently, Pal and Talukdar [11] analyzed the heat and mass transfer in boundary layer slip flow past a vertical permeable plate with thermal radiation and chemical reaction. They reported analytical solution and found that the effect of thermal radiation and chemical reaction decreases the velocity and concentration profiles. Chamkha [12] obtained the numerical solution for the problem of heat and mass transfer by the steady flow of an electrically conducting fluid over a uniformly moving vertical surface in the presence of firstorder chemical reaction. Later, the heat and mass transfer of a viscoelastic fluid in a fixed plane with radiation and chemical reaction effect was investigated by Uwanta and Omokhuale [13], and they found that temperature decreases with the increase in Prandtl number and concentration decreases with the increase in chemical reaction parameter. Other examples dealing with radiation and chemical reaction effects can be found in $[14,15]$.

The study of fluid flow with subject to convective boundary condition plays an important role in several engineering and industrial processes like transpiration cooling process and material drying. Therefore several authors [16-19] have shown their interest in obtaining solutions subject to convective boundary condition. Interestingly, in all of the above investigations the Hall term has been ignored in applying Ohms law as it has no marked effect for small and moderate values of the magnetic field. However, the current trend for the application of magnetohydrodynamics is towards a strong magnetic field, so that the influence of electromagnetic force is noticeable [20]. Under these conditions, the Hall current is important and it has a marked effect on the magnitude and direction of the current density and magnetic force term. With this understanding, very recently, Hayat and Nawaz [21] have studied the effect of Hall current on the mixed convection flow of a second grade fluid in the presence of Soret and Dufour effects.

Previous studies of the flow with heat and mass transfer have focused mainly on a flat wall or a regular channel. It is necessary to study the flow, heat and mass transfer in an irregular channel because such flows find applications in different areas such as transpiration cooling of reentry vehicles and rocket boosters, crosshatching on ablative surfaces, and film vaporization in combustion chambers. In view of these applications, Das [22] analyzed the viscous incompressible fluid confined between a long vertical wavy wall and a parallel flat wall. The effects of time-dependent pulsatile flow and heat transfer of a Newtonian fluid (blood) through a vertical annulus with mild constriction on the outer wall while the inner wall represents the catheter tube were studied by Abd elmaboud and Mekheimer [23]. Many authors like in [24-27] have analyzed the fluid flow in wavy-walled channels.

Keeping all these facts in mind, in this work the effect of radiation on unsteady laminar flow with heat and mass transfer of an electrically conducting, chemically reactive viscoelastic fluid in irregular channel with subject to convective boundary condition has been investigated. The perturbation technique is employed to solve governing coupled nonlinear partial differential equations.

\section{Walters B Liquid Model}

The constitutive equations for the rheological equation of the state for the visco-elastic fluid (Walters liquid B) are

$$
\begin{gathered}
p_{i k}=-p g_{i k}+p_{i k}^{*}, \\
p_{i k}^{*}=2 \int_{-\infty}^{t} \psi\left(t-t^{*}\right) e_{i k}^{(1)}\left(t^{*}\right) d t^{*}
\end{gathered}
$$

where

$$
\psi\left(t-t^{*}\right)=\int_{0}^{\infty} \frac{N(\tau)}{\tau} e^{\left(-\left(t-t^{*}\right) / \tau\right)} d \tau .
$$

In the above equations $p_{i k}$ is the stress tensor, $p$ is an arbitrary isotropic pressure, $g_{i k}$ is the metric tensor of a fixed coordinate system $x^{i}$, and $e_{i k}^{(1)}$ is the rate of strain tensor. $N(\tau)$ is the distribution function of relaxation time $(\tau)$. Equation (2) can be put in the following generalized form which is valid for all types of motion and stress; it was shown by Walters [28]:

$$
p_{i k}^{*}(x, t)=2 \int_{-\infty}^{t} \psi\left(t-t^{*}\right) \frac{\partial x^{i}}{\partial x_{m}^{*}} \frac{\partial x^{k}}{\partial x_{r}^{*}} e_{m r}^{(1)}\left(x^{*} t^{*}\right) d t^{*},
$$

where $x^{* i}=x^{* i}\left(x, t, t^{*}\right)$ is the position at the time $t^{*}$ of the element which is instantaneously at the position $x^{i}$ at the time $t$. The fluid with equations of state (1) and (4) has been designated as liquid " $B$." In the case of liquids with short memories, that is, short relaxation times, the above equation of state can be written in the following simplified form:

$$
p_{i k}^{*}(x, t)=2 \eta_{0} e_{i k}^{(1)}-2 k_{0} \frac{\partial e_{i k}^{(1)}}{\partial t},
$$

where $\eta_{0}=\int_{0}^{\infty} N(\tau) d \tau$ is the limiting viscosity at small rates of shear. $k_{0}=\int_{0}^{\infty} \tau N(\tau) d \tau$ is the Walters-B viscoelasticity parameter, and $\partial / \partial t$ denotes the convected time derivative. This rheological model is very versatile and robust and provides a relatively simple mathematical formulation which is easily incorporated into boundary layer theory for engineering applications.

\section{Mathematical Formulation and Solution}

Consider an unsteady flow, heat and mass transfer of an electrically conducting, incompressible, chemically reacting viscoelastic fluid between a vertical long wavy wall under the influence of uniform transverse magnetic field of strength $B_{0}$ and exponentially decreasing pressure gradient. A parallel flat wall saturated with porous medium is placed in the plane $y=0$ of a coordinate system. The $x$-axis is taken in the direction along the channel which is set in motion, and the $y$-axis is taken perpendicular to it. The inclusion of the Hall currents give's rise to the Lorentz force in $z$-direction, which induces a crossflow in that direction. The $z$-axis is assumed to be normal to the $x-y$ plane which is as shown in Figure 1 .

The flow field is exposed to the influence of thermal and mass buoyancy effects, Hall effect, thermal radiation, heat absorption, and first order chemically reactive species. The 


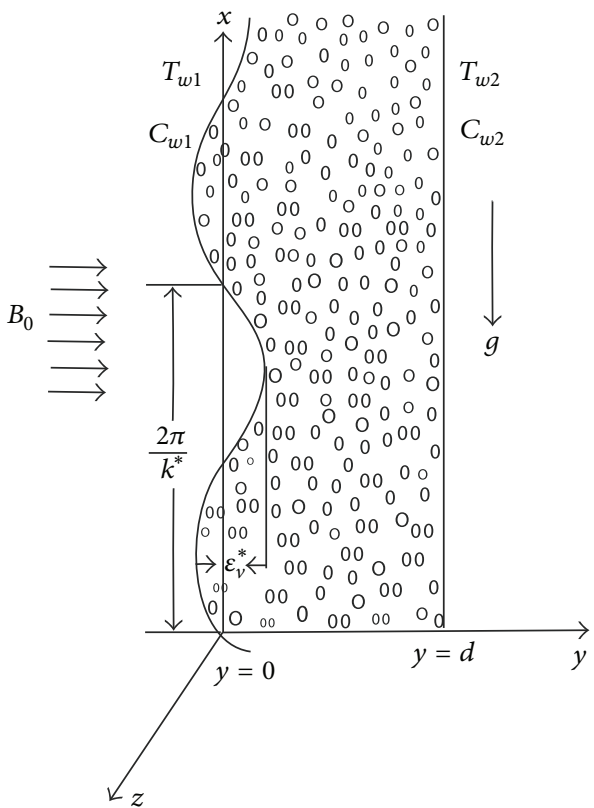

FIGURE 1: The geometry of the problem.

magnetic Reynolds number is assumed to be small enough so that the induced magnetic field is negligible. The wavy wall $\left(y=\varepsilon_{v}^{*} \cos \left(\lambda^{*} x\right)\right)$ maintains a temperature $T_{w 1}$ which represents the convective boundary condition $-k(\partial T / \partial y)=$ $h_{f}\left(T_{1}-T+\left(T_{2}-T_{1}\right) \varepsilon e^{-n t}\right)$ and concentration $C_{w 1}=C_{1}+\left(C_{2}-\right.$ $\left.C_{1}\right) \varepsilon e^{-n t}$, respectively. It is assumed that the convective heat exchange with the wall temperature at the channel surface follows Newton's law of cooling.

The equations governing the flow are in vector form.

\section{Momentum Equation}

$$
\begin{aligned}
\frac{\partial \vec{q}}{\partial t}+(\vec{q} \cdot \nabla) \vec{q}= & -\frac{1}{\rho} \nabla P+\nu \nabla^{2} \vec{q}+\nabla \cdot p_{i k}+\frac{1}{\rho}(\vec{J} \times \vec{B}) \\
& -\frac{v}{k(t)} \vec{q}+g \beta_{T}\left(T-T_{1}\right)+g \beta_{C}\left(C-C_{1}\right) .
\end{aligned}
$$

\section{Continuity Equation}

$$
\nabla \cdot \vec{q}=0
$$

\section{Energy Equation}

$$
\frac{\partial T}{\partial t}+(\vec{q} \cdot \nabla) T=\frac{k}{\rho C_{p}} \nabla^{2} T-\frac{1}{\rho C_{p}} \nabla q_{r}-\frac{Q_{T}}{\rho C_{p}}\left(T-T_{1}\right) .
$$

\section{Mass Diffusion Equation}

$$
\frac{\partial C}{\partial t}+(\vec{q} \cdot \nabla) C=D \nabla^{2} C-K_{R}\left(C-C_{1}\right),
$$

where $\rho$ is the density of the fluid, $\nu$ is the kinematic viscosity of the fluid, $v_{1}$ is the kinematic viscoelasticity of the fluid, $\vec{q}(u, v, w)$ is the velocity vector, $u, v$, and $w$ are the velocity components along $x, y$, and $z$ directions, respectively, $\vec{B}\left(0, B_{0}, 0\right)$ is the magnetic induction, $\vec{J}\left(J_{x}, J_{y}, J_{z}\right)$ is the current density vector, $P$ is the pressure, $p_{i k}$ is the stress tensor, $g$ is the acceleration due to gravity, $\beta_{T}$ is the thermal expansion coefficient, $\beta_{C}$ is the concentration expansion coefficient, $T$ is the temperature of the fluid, $C$ is the concentration of the fluid, $k$ is the thermal conductivity, $C_{p}$ is the specific heat of the fluid at constant pressure, $q_{r}$ is the radiative heat flux, $Q_{T}$ is the heat absorption coefficient, $K_{R}$ is the chemical reaction parameter, $T_{1}$ and $T_{2}$ are wall temperatures, $C_{1}$ and $C_{2}$ are wall concentrations, and $t$ is the time. If the Hall term is retained, the current density $\vec{J}$ from the generalized Ohm's law is given by

$$
\vec{J}+\frac{\omega_{e} \tau_{e}}{B_{0}}(\vec{J} \times \vec{B})=\sigma\left(\vec{E}+\vec{q} \times \vec{B}+\frac{\nabla P_{e}}{e \eta_{e}}\right),
$$

where $\omega_{e}$ is the electron frequency, $\tau_{e}$ is the electron collision time, $e$ is the electron charge, $\eta_{e}$ is the number density of electron, $P_{e}$ is the electron pressure, $\sigma$ is the electric conductivity, and $E$ is the electric field. In (10) ion slip, thermoelectric effect, and polarization effects are neglected. Solving (10) for $\vec{J}$, it gives

$$
\vec{J} \times \vec{B}=\frac{\sigma B_{0}^{2}}{\left(1+m^{2}\right)}[-(m w+u) \hat{i}+(m u-w) \hat{k}]
$$

where $m=\omega_{e} \tau_{e}$ is the Hall parameter. Under the above stated assumptions and usual Boussinesq approximation, the governing equations in their component form are reduced to:

$$
\begin{gathered}
\frac{\partial u}{\partial t}=-\frac{1}{\rho} \frac{\partial p}{\partial x}+\nu \frac{\partial^{2} u}{\partial y^{2}}-\nu_{1} \frac{\partial^{3} u}{\partial y^{2} \partial t}-\frac{\sigma B_{0}^{2}}{\rho\left(1+m^{2}\right)}(m w+u) \\
-\frac{v}{k(t)} u+g \beta_{T}\left(T-T_{1}\right)+g \beta_{C}\left(C-C_{1}\right) \\
\frac{\partial w}{\partial t}=v \frac{\partial^{2} w}{\partial y^{2}}-\nu_{1} \frac{\partial^{3} w}{\partial y^{2} \partial t}+\frac{\sigma B_{0}^{2}}{\rho\left(1+m^{2}\right)}(m u-w)-\frac{v}{k(t)} w \\
\rho C_{p} \frac{\partial T}{\partial t}=k \frac{\partial^{2} T}{\partial y^{2}}-\frac{\partial q_{r}}{\partial y}-Q_{T}\left(T-T_{1}\right), \\
\frac{\partial C}{\partial t}=D \frac{\partial^{2} C}{\partial y^{2}}-K_{R}\left(C-C_{1}\right) .
\end{gathered}
$$

The suitable initial and boundary conditions for the present problem are

$$
\begin{gathered}
t=0: \quad u=0, \quad w=0, \quad T=T_{1}, \quad C=C_{1}, \\
\text { for } y \in\left(\varepsilon_{v} \cos \left(\lambda^{*} x\right), d\right),
\end{gathered}
$$




$$
\begin{aligned}
t>0: & u=0, \quad w=0, \\
& -k \frac{\partial T}{\partial y}=h_{f}\left(T_{1}-T+\varepsilon\left(T_{2}-T_{1}\right) e^{-n t}\right), \\
& C=C_{1}+\varepsilon\left(C_{2}-C_{1}\right) e^{-n t} \quad \text { at } y=\varepsilon_{v} \cos (\lambda x), \\
: & u=0, \quad w=0, \\
& -k \frac{\partial T}{\partial y}=h_{f}\left(T_{2}-T+\varepsilon\left(T_{2}-T_{1}\right) e^{-n t}\right), \\
C & =C_{2}+\varepsilon\left(C_{2}-C_{1}\right) e^{-n t} \quad \text { at } y=d,
\end{aligned}
$$

where $n$ is the positive real constant, $\varepsilon_{v}^{*}$ is the amplitude parameter, $h_{f}$ is the heat transfer coefficient, $d$ is the dimensional width of the channel, and $\varepsilon$ is a small positive constant $(\varepsilon \ll 1)$.

Using the relation in [29], the radiative heat flux $\left(q_{r}\right)$ for the optically thin nongray gas near equilibrium is given by

$$
\frac{\partial q_{r}}{\partial y}=4 I^{\prime}\left(T-T_{1}\right), \quad I^{\prime}=\int_{0}^{\infty} K_{\lambda_{1} w} \frac{\partial e_{b \lambda_{1}}}{\partial T} d \lambda_{1},
$$

where $q_{r}$ is the radiative heat flux, $K_{\lambda_{1} w}$ is the radiation absorption coefficient at the wall, and $e_{b \lambda_{1}}$ is Plank's constant.

The permeability of the porous medium is assumed to be of the following form:

$$
k(t)=k_{0}^{+}\left(1+\varepsilon e^{-n t}\right),
$$

where $k_{0}$ is the constant permeability of the medium.

Now we introduce the following non dimensional quantities:

$$
\begin{array}{r}
\left(x^{*}, y^{*}\right)=\frac{(x, y)}{d}, \quad u^{*}=\frac{u}{u_{0}}, \quad w^{*}=\frac{w}{u_{0}}, \\
t^{*}=\frac{v t}{d^{2}}, \quad n^{*}=\frac{n d^{2}}{v} \quad \varepsilon_{v}^{*}=\frac{\varepsilon_{v}}{d}, \\
p^{*}=\frac{p d}{v u_{0}}, \quad T^{*}=\frac{T-T_{1}}{T_{2}-T_{1}}, \quad C^{*}=\frac{C-C_{1}}{C_{2}-C_{1}} .
\end{array}
$$

Using (14), (15), and (16) and then introducing complex velocity $\psi^{*}=u^{*}+i w^{*}$ into (12) one can get (dropping the asterisk ${ }^{*}$ )

$$
\begin{gathered}
\frac{\partial \psi}{\partial t}=-\frac{1}{\rho} \frac{\partial p}{\partial x}+\frac{\partial^{2} \psi}{\partial y^{2}}-E \frac{\partial^{3} \psi}{\partial y^{2} \partial t}-\frac{M^{2}(1-i m)}{\left(1+m^{2}\right)} \psi \\
-\frac{1}{k_{0}\left(1+\varepsilon e^{-n t}\right)} \psi+\mathrm{Gr} T+\mathrm{GcC}, \\
\operatorname{Pr} \frac{\partial T}{\partial t}=\frac{\partial^{2} T}{\partial y^{2}}-R T-\alpha_{T} T, \\
\operatorname{Sc} \frac{\partial C}{\partial t}=\frac{\partial^{2} C}{\partial y^{2}}-\mathrm{KrSc} C,
\end{gathered}
$$

where $E=v_{1} / d^{2}$ is the viscoelastic parameter, $M=$ $B_{0} d \sqrt{\sigma_{e} / \nu \rho}$ is the magnetic parameter, $\mathrm{Gr}=g \beta_{T}\left(T_{2}-\right.$ $\left.T_{1}\right) d^{2} / \nu u_{0}$ is the thermal Grashof number, Gc $=g \beta_{C}\left(C_{2}-\right.$ $\left.C_{1}\right) d^{2} / \nu u_{0}$ is the solutal Grashof number, $\operatorname{Pr}=\mu C_{p} / k$ is the Prandtl number, $R=4 I^{\prime} d^{2} / k$ is the radiation parameter, $k_{0}=k_{0}^{+} / d^{2}$ is the permeability parameter, $\alpha_{T}=Q_{T} d^{2} / k$ is the heat absorption parameter, $\mathrm{Sc}=v / d$ is the Schmidt number, and $\mathrm{Kr}=h^{2} K_{R} / \nu$ is the chemical reaction parameter.

The corresponding initial and boundary conditions (16) in dimensionless form are

$$
\begin{gathered}
t=0: \psi=0, \quad T=0, \quad C=0, \quad \text { for } y \in(h, 1), \\
t>0: \quad \psi=0, \quad \frac{\partial T}{\partial y}=B_{i}\left(T-\varepsilon e^{-n t}\right), \quad C=\varepsilon e^{-n t} \\
\text { at } y=h, \\
: \psi=0, \quad \frac{\partial T}{\partial y}=B_{i}\left(T-1-\varepsilon e^{-n t}\right), \\
C=1+\varepsilon e^{-n t} \quad \text { at } y=1,
\end{gathered}
$$

where $B_{i}=h_{f} d / k$ is the Biot number, $h=\varepsilon_{v} \cos (\lambda x)$, and $\lambda=\lambda^{*} d$ is the frequency parameter of the wavy wall.

Equations (17) represent the set of partial differential equations that cannot be solved in the closed form. However, these equations can be solved analytically after being reduced to set of ordinary differential equations in dimensionless form. Thus, to this end we can represent the velocity $(\psi)$, temperature $(T)$, and concentration $(C)$ in terms of power of $\varepsilon(\varepsilon \ll 1)$ and as follows:

$$
\begin{aligned}
& \psi(y, t)=\psi_{0}(y)+\varepsilon \psi_{1}(y, t) e^{-n t}+O\left(\varepsilon^{2}\right), \\
& T(y, t)=T_{0}(y)+\varepsilon T_{1}(y, t) e^{-n t}+O\left(\varepsilon^{2}\right), \\
& C(y, t)=C_{0}(y)+\varepsilon C_{1}(y, t) e^{-n t}+O\left(\varepsilon^{2}\right) .
\end{aligned}
$$

Let us assume the pressure gradient is of the form $-(1 / \rho)(\partial p / \partial x)=A+\varepsilon B e^{-n t}$, where $A$ and $B$ are constants. Now by substituting (19) into (17), then equating harmonic and nonharmonic terms and neglecting the higher-order terms of $\varepsilon$, then we get the following set of equations:

$$
\begin{gathered}
\psi_{0}^{\prime \prime}(y)-\left(\frac{M^{2}(1-i m)}{1+m^{2}}+\frac{1}{k_{0}}\right) \psi_{0}(y) \\
=-A-\mathrm{GrT}_{0}-\mathrm{GcC}_{0}, \\
(1+n E) \psi_{1}^{\prime \prime}(y, t)-\left[\frac{M^{2}(1-i m)}{1+m^{2}}+\frac{1}{k_{0}}-n\right] \psi_{1}(y, t) \\
=-B-\mathrm{GrT}_{1}-\mathrm{GcC}_{1}, \\
T_{0}^{\prime \prime}(y)-\left(R+\alpha_{T}\right) T_{0}(y)=0, \\
T_{1}^{\prime \prime}(y, t)-\left(R+\alpha_{T}-n \operatorname{Pr}\right) T_{1}(y, t)=0,
\end{gathered}
$$


ISRN Thermodynamics

5

$$
\begin{gathered}
C_{0}^{\prime \prime}(y)-\operatorname{KrSc}_{0}(y)=0, \\
C_{1}^{\prime \prime}(y, t)-\operatorname{Sc}(\mathrm{Kr}-n) C_{1}(y, t)=0 .
\end{gathered}
$$

The corresponding boundary conditions will become

$$
\begin{gathered}
\psi_{0}=0, \quad T_{0}^{\prime}=B_{i} T_{0}, \quad C_{0}=0, \\
\psi_{1}=0, \quad T_{1}^{\prime}=B_{i}\left(T_{1}-1\right), \quad C_{1}=1 \quad \text { at } y=h, \\
\psi_{0}=0, \quad T_{0}^{\prime}=B_{i}\left(T_{0}-1\right), \quad C_{0}=1, \\
\psi_{1}=0, \quad T_{1}^{\prime}=B_{i}\left(T_{1}-1\right), \quad C_{1}=1 \quad \text { at } y=+1,
\end{gathered}
$$

where prime $\left({ }^{\prime}\right)$ denotes the differentiation with respect to $y$. The solutions of (20) subject to the boundary conditions (21) are

$$
\begin{aligned}
C(y, t)= & A_{1} e^{\beta_{1} y}+A_{2} e^{\beta_{2} y}+\varepsilon\left(A_{3} e^{\beta_{3} y}+A_{4} e^{\beta_{4} y}\right) e^{-n t} \\
T(y, t)= & A_{5} e^{\beta_{5} y}+A_{6} e^{\beta_{6} y}+\varepsilon\left(A_{7} e^{\beta_{7} y}+A_{8} e^{\beta_{8} y}\right) e^{-n t} \\
\psi(y, t)= & A_{9} e^{\beta_{1} y}+A_{10} e^{\beta_{2} y}+A_{11} e^{\beta_{5} y}+A_{12} e^{\beta_{6} y} \\
& +A_{13}+A_{14} e^{\beta_{9} y}+A_{15} e^{\beta_{10} y} \\
& +\varepsilon\left(A_{16} e^{\beta_{3} y}+A_{17} e^{\beta_{4} y}+A_{18} e^{\beta_{7} y}+A_{19} e^{\beta_{8} y}\right. \\
& \left.+A_{20}+A_{21} e^{\beta_{11} y}+A_{22} e^{\beta_{12} y}\right) e^{-n t} .
\end{aligned}
$$

On separating real and imaginary parts in (24), we get the axial velocity $(u)$ and transverse velocity $(w)$ profiles.

Skin Friction. The skin friction $\left(\tau=\tau_{1}+i \tau_{2}\right)$ expression for viscoelastic fluid at a wavy wall $y=h$ is given by

$$
\begin{aligned}
\tau= & \left(\frac{\partial \psi}{\partial y}\right)_{y=h} \\
= & \beta_{1} A_{9} e^{\beta_{1} h}+\beta_{2} A_{10} e^{\beta_{2} h}+\beta_{5} A_{11} e^{\beta_{5} h} \\
& +\beta_{6} A_{12} e^{\beta_{6} h}+\beta_{9} A_{14} e^{\beta_{9} h}+\beta_{10} A_{15} e^{\beta_{10} h} \\
& +\varepsilon\left(\beta_{3} A_{16} e^{\beta_{3} h}+\beta_{4} A_{17} e^{\beta_{4} h}+\beta_{7} A_{18} e^{\beta_{7} h}+\beta_{8} A_{19} e^{\beta_{8}}\right. \\
& \left.\quad+\beta_{11} A_{21} e^{\beta_{11} h}+\beta_{12} A_{22} e^{\beta_{12} h}\right) e^{-n t},
\end{aligned}
$$

where $\tau_{1}$ and $\tau_{2}$ are the axial and transverse components of the skin friction, respectively.

Nusselt Number. The Nusselt number $(\mathrm{Nu})$ expression for visco-elastic fluid at a wavy wall $y=h$ is given by

$$
\begin{aligned}
\mathrm{Nu}=\left(\frac{\partial T}{\partial y}\right)_{y=h}= & \beta_{5} A_{5} e^{\beta_{5} h}+\beta_{6} A_{6} e^{\beta_{6} h} \\
& +\varepsilon\left(\beta_{7} A_{7} e^{\beta_{7} h}+\beta_{8} A_{8} e^{\beta_{8} h}\right) e^{-n t} .
\end{aligned}
$$

Sherwood Number. The Sherwood number (Sh) expression for visco-elastic fluid at a wavy wall $y=h$ is given by

$$
\begin{aligned}
\text { Sh }=\left(\frac{\partial C}{\partial y}\right)_{y=h}= & \beta_{1} A_{1} e^{\beta_{1} h}+\beta_{2} A_{2} e^{\beta_{2} h} \\
& +\varepsilon\left(\beta_{3} A_{3} e^{\beta_{3} h}+\beta_{4} A_{4} e^{\beta_{4} h}\right) e^{-n t},
\end{aligned}
$$

where

$$
\begin{gathered}
\beta_{1}=-\beta_{2}=\sqrt{\mathrm{KrSc}}, \quad \beta_{3}=-\beta_{4}=\sqrt{\mathrm{Sc}(\mathrm{Kr}-n)}, \\
\beta_{5}=-\beta_{6}=\sqrt{R+\alpha_{T}},
\end{gathered}
$$

$$
\begin{gathered}
\beta_{11}=-\beta_{12}=P=\sqrt{\frac{1}{(1+n E)}\left[\frac{M^{2}(1-i m)}{1+m^{2}}+\frac{1}{k_{0}}-n\right]}, \\
\beta_{9}=-\beta_{10}=N=\sqrt{\left(\frac{M^{2}(1-i m)}{1+m^{2}}+\frac{1}{k_{0}}\right)}, \\
\beta_{7}=-\beta_{8}=\sqrt{R+\alpha_{T}-n P r},
\end{gathered}
$$$$
\begin{array}{ll}
A_{1}=\frac{e^{\beta_{2} h}}{e^{\beta_{1}} e^{\beta_{2} h}-e^{\beta_{1} h} e^{\beta_{2}}}, & A_{2}=\frac{e^{\beta_{1} h}}{e^{\beta_{2}} e^{\beta_{1} h}-e^{\beta_{2} h} e^{\beta_{1}}}, \\
A_{3}=\frac{e^{\beta_{4} h}-e^{\beta_{4}}}{e^{\beta_{3}} e^{\beta_{4} h}-e^{\beta_{3} h} e^{\beta_{4}}}, & A_{4}=\frac{e^{\beta_{3} h}-e^{\beta_{3}}}{e^{\beta_{4}} e^{\beta_{3} h}-e^{\beta_{4} h} e^{\beta_{3}}},
\end{array}
$$

$$
\begin{aligned}
& A_{5}=\frac{B_{i} e^{\beta_{6} h}}{\left(\beta_{5}-B_{i}\right)\left(e^{\beta_{6}} e^{\beta_{5} h}-e^{\beta_{6} h} e^{\beta_{5}}\right)}, \\
& A_{6}=\frac{B_{i} e^{\beta_{5} h}}{\left(\beta_{6}-B_{i}\right)\left(e^{\beta_{5}} e^{\beta_{6} h}-e^{\beta_{5} h} e^{\beta_{6}}\right)},
\end{aligned}
$$$$
A_{7}=\frac{B_{i}\left(e^{\beta_{8} h}-e^{\beta_{8}}\right)}{\left(\beta_{7}-B_{i}\right)\left(e^{\beta_{8}} e^{\beta_{7} h}-e^{\beta_{8} h} e^{\beta_{7}}\right)},
$$$$
A_{8}=\frac{B_{i}\left(e^{\beta_{7} h}-e^{\beta_{7}}\right)}{\left(\beta_{8}-B_{i}\right)\left(e^{\beta_{7}} e^{\beta_{8} h}-e^{\beta_{7} h} e^{\beta_{8}}\right)},
$$$$
A_{9}=\frac{\mathrm{Gc} A_{1}}{N^{2}-\beta_{1}^{2}}, \quad A_{10}=\frac{\mathrm{Gc} A_{2}}{N^{2}-\beta_{2}^{2}},
$$

$$
\begin{aligned}
A_{11}= & \frac{\mathrm{Gr} A_{5}}{N^{2}-\beta_{5}^{2}}, \quad A_{12}=\frac{\mathrm{Gr} A_{6}}{N^{2}-\beta_{6}^{2}}, \quad A_{13}=\frac{A}{N^{2}}, \\
A_{14}^{*}= & A_{9}\left(e^{\beta_{1}} e^{\beta_{10} h}-e^{\beta_{1} h} e^{\beta_{10}}\right)+A_{10}\left(e^{\beta_{2}} e^{\beta_{10} h}-e^{\beta_{2} h} e^{\beta_{10}}\right) \\
& +A_{11}\left(e^{\beta_{5}} e^{\beta_{10} h}-e^{\beta_{5} h} e^{\beta_{10}}\right) \\
& +A_{12}\left(e^{\beta_{6}} e^{\beta_{10} h}-e^{\beta_{6} h} e^{\beta_{10}}\right)+A_{13}\left(e^{\beta_{10} h}-e^{\beta_{10}}\right) \\
A_{15}^{*}= & A_{9}\left(e^{\beta_{1}} e^{\beta_{9} h}-e^{\beta_{1} h} e^{\beta_{9}}\right)+A_{10}\left(e^{\beta_{2}} e^{\beta_{9} h}-e^{\beta_{2} h} e^{\beta_{9}}\right) \\
& +A_{11}\left(e^{\beta_{5}} e^{\beta_{9} h}-e^{\beta_{5} h} e^{\beta_{9}}\right) \\
& +A_{12}\left(e^{\beta_{6}} e^{\beta_{9} h}-e^{\beta_{6} h} e^{\beta_{9}}\right)+A_{13}\left(e^{\beta_{9} h}-e^{\beta_{9}}\right),
\end{aligned}
$$




$$
\begin{aligned}
& A_{14}=\frac{A_{14}^{*}}{e^{\beta_{10}} e^{\beta_{9} h}-e^{\beta_{10} h} e^{\beta_{9}}}, \quad A_{15}=\frac{A_{15}^{*}}{e^{\beta_{10} h} e^{\beta_{9}}-e^{\beta_{10}} e^{\beta_{9} h}}, \\
& A_{16}=\frac{\mathrm{Gc}_{3}}{\left(P^{2}-\beta_{3}^{2}\right)(1+n E)}, \quad A_{17}=\frac{\mathrm{Gc}_{4}}{\left(P^{2}-\beta_{4}^{2}\right)(1+n E)}, \\
& A_{18}=\frac{\mathrm{Gr} A_{7}}{\left(P^{2}-\beta_{7}^{2}\right)(1+n E)}, \\
& A_{19}=\frac{G r A_{8}}{\left(P^{2}-\beta_{8}^{2}\right)(1+n E)}, \quad A_{20}=\frac{B}{P^{2}(1+n E)}, \\
& A_{21}^{*}=A_{16}\left(e^{\beta_{12}} e^{\beta_{3} h}-e^{\beta_{12} h} e^{\beta_{3}}\right)+A_{17}\left(e^{\beta_{12}} e^{\beta_{4} h}-e^{\beta_{12} h} e^{\beta_{4}}\right) \\
& +A_{18}\left(e^{\beta_{12}} e^{\beta_{7} h}-e^{\beta_{12} h} e^{\beta_{7}}\right) \\
& +A_{19}\left(e^{\beta_{12}} e^{\beta_{8} h}-e^{\beta_{12} h} e^{\beta_{8}}\right)+A_{20}\left(e^{\beta_{12}}-e^{\beta_{12} h}\right), \\
& A_{22}^{*}=A_{16}\left(e^{\beta_{11}} e^{\beta_{3} h}-e^{\beta_{11} h} e^{\beta_{3}}\right)+A_{17}\left(e^{\beta_{11}} e^{\beta_{4} h}-e^{\beta_{11} h} e^{\beta_{4}}\right) \\
& +A_{18}\left(e^{\beta_{11}} e^{\beta_{7} h}-e^{\beta_{11} h} e^{\beta_{7}}\right) \\
& +A_{19}\left(e^{\beta_{11}} e^{\beta_{8} h}-e^{\beta_{11} h} e^{\beta_{8}}\right)+A_{20}\left(e^{\beta_{11}}-e^{\beta_{11} h}\right), \\
& A_{21}=\frac{A_{21}^{*}}{e^{\beta_{11}} e^{\beta_{12} h}-e^{\beta_{11} h} e^{\beta_{12}}}, \quad A_{22}=\frac{A_{22}^{*}}{e^{\beta_{11} h} e^{\beta_{12}}-e^{\beta_{11}} e^{\beta_{12} h}} .
\end{aligned}
$$

\section{Results and Discussion}

A hydromagnetic flow, heat and mass transfer of a chemically reacting viscoelastic fluid in an irregular channel with convective boundary condition are investigated in the presence of heat absorption, thermal radiation, and Hall effect. The governing partial differential equations are solved analytically using perturbation technique. In order to get a physical insight of the problem, we have written a MATLAB program to perform a parametric study on different flow fields versus $y$ for the different values of flow parameters. The parametric study is carried out for both the cases corresponding to cooling $(\mathrm{Gr}>0)$ and heating $(\mathrm{Gr}<0)$ of the plate by free convection currents. The results of this parametric study are presented graphically and are shown in Figures 2-23. During the numerical computations of velocity and temperature fields, the value of Prandtl number is taken as 3 which physically corresponds to Freon. Freon represents several different chlorofluorocarbons (CFCs) which are used in engineering and industrial applications.

Figures 2 and 3 indicate the effect of magnetic parameter, in both $\mathrm{Gr}<0$ and $\mathrm{Gr}>0$ cases on axial velocity and transverse velocity profile, respectively. We can see that in Figure 2 with increasing magnetic parameter, the axial velocity profile decreases in both the cases $(\mathrm{Gr}<0$ and $\mathrm{Gr}>0)$. Physically, it is justified because transverse magnetic field yields to create a drag-like force called Lorentz force to resist the flow so magnetic field parameter slows down the flow and causes the axial velocity to decrease. But this trend is opposite for transverse velocity profiles in both the cases $(\mathrm{Gr}<0$ and $\mathrm{Gr}>0)$.

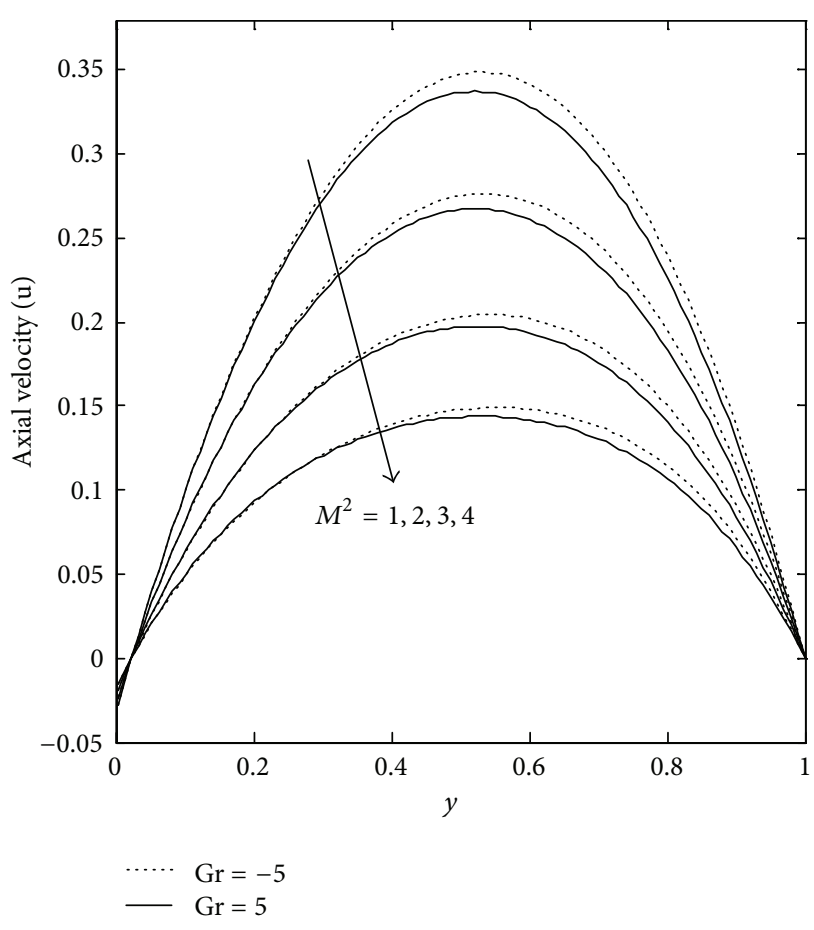

FIGURE 2: Variation of axial velocity profile for the different values of $M^{2}$.

In Figure 4, it is shown that with the increase in viscoelastic parameter, the axial velocity profile descends in both the cases. Because viscoelasticity introduces tensile stress the fluid motion contracts transversely, and hence axial velocity retards. It is also observed that the velocity of Newtonian fluid $(E=0)$ is higher than that of non-Newtonian fluid $(E \neq 0)$ in both cases. The secondary velocity field is enhanced due to increase in visco-elastic parameter in both cases, which is depicted in Figure 5.

Figures 6 to 9 elucidate the inclusion of the buoyancy effects. The presence of the buoyancy effects complicates the problem, by coupling of the flow problem with thermal and mass problem. Figures 6 and 7 have been plotted to show the effect of the thermal Grashof number on primary and secondary velocity profiles, respectively. The values of Grashof number $\mathrm{Gr}=-8,-6,-4,-2,2,4,6,8$ are chosen. Here the negative and positive values of Grashof number physically represent heating and cooling of the plate, respectively. Hence, it is observed from Figures 6 and 7 that an increase in the thermal Grashof number leads to increase in both the axial and transverse velocity profiles due to the enhancement of buoyancy forces. Actually, the thermal Grashof number signifies the relative importance of buoyancy force to the viscous hydrodynamic force. The increase in Grashof number indicates small viscous effects in momentum equation and consequently causes increase in the velocity profiles. With the increase in the solutal Grashof number, the axial and transverse velocity profiles increase in both cases $(\mathrm{Gr}<0$ and Gr $>0$ ) as shown in Figures 8 and 9, respectively. The solutal Grashof number defines the ratio of species buoyancy force 


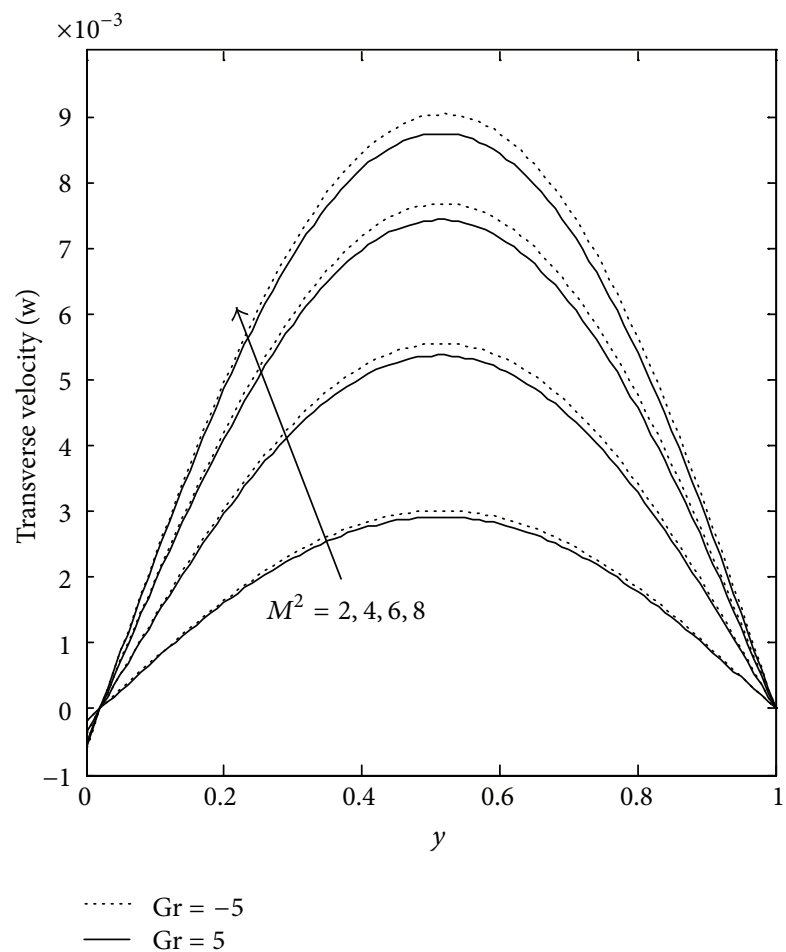

FIGURE 3: Variation of transverse velocity profile for the different values of $M^{2}$.

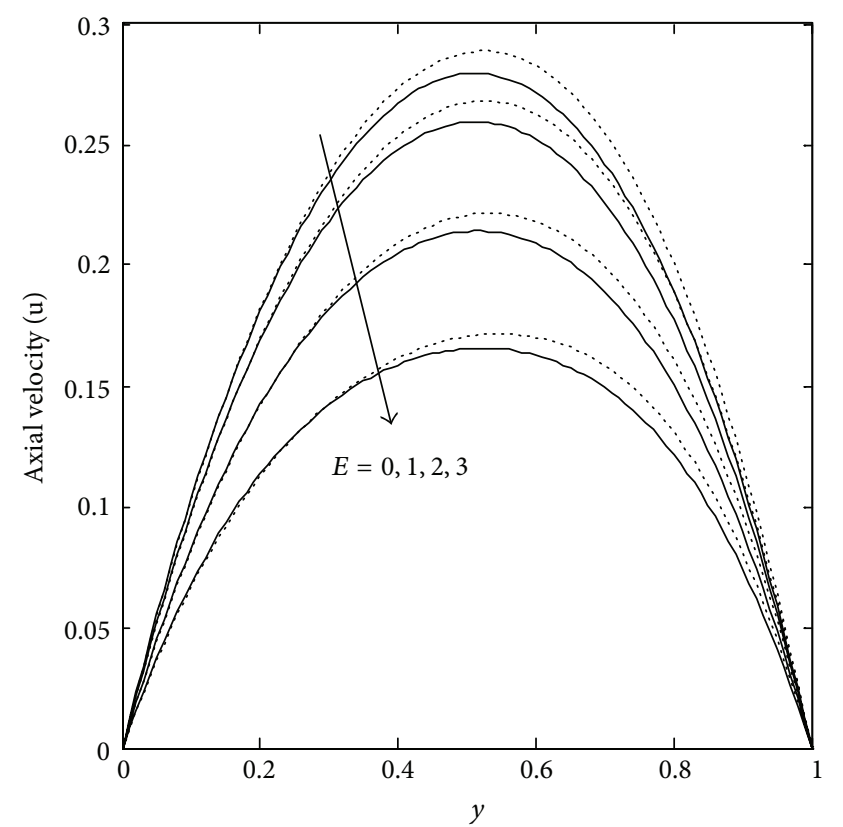

Gr $=-5$

- $\mathrm{Gr}=5$

FIGURE 4: Variation of axial velocity profile for the different values of $E$.

to the viscous hydrodynamic force, so as to expect the fluid velocity increases.

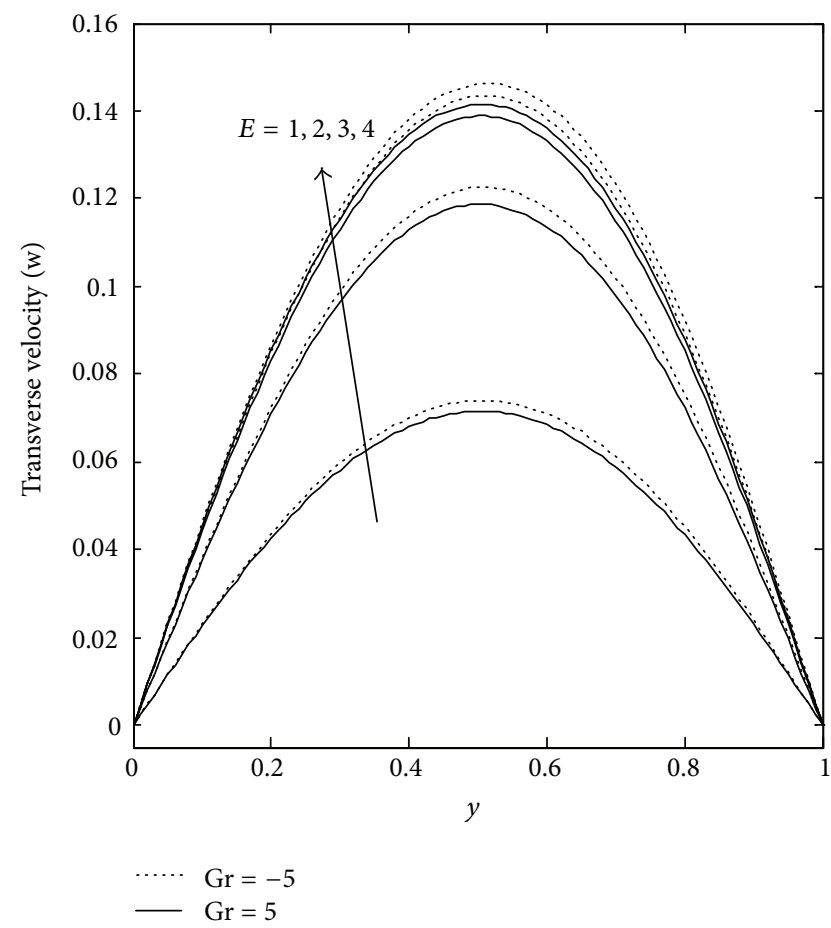

FIGURE 5: Variation of transverse velocity profile for the different values of $E$.

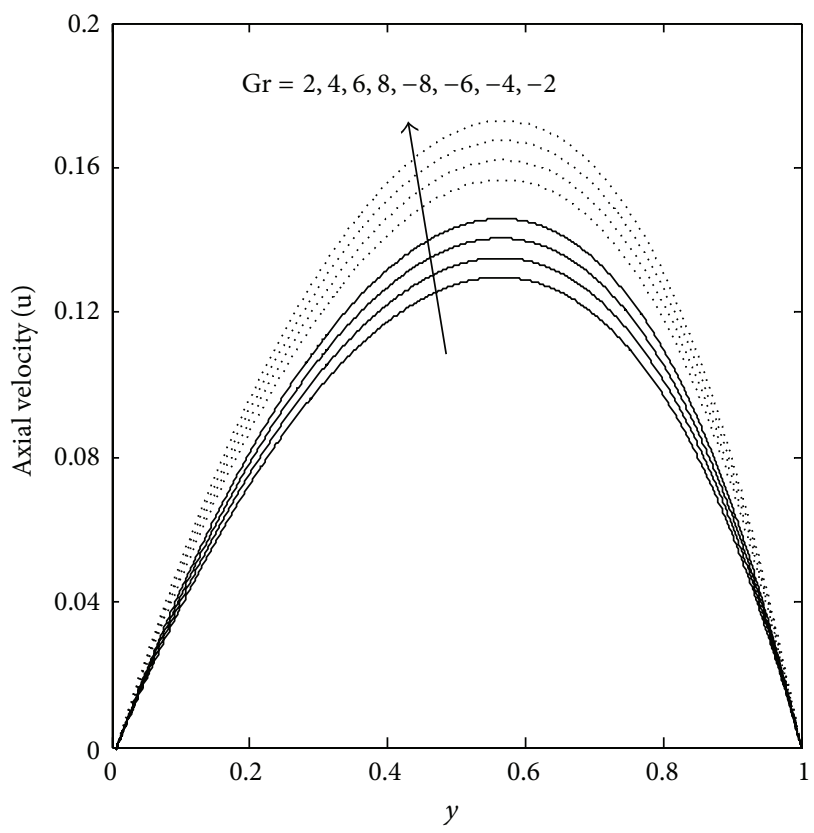

FIGURE 6: Variation of axial velocity profile for the different values of Gr.

One can see that in Figures 10 and 11 with increasing Hall parameter, the axial and transverse velocity profiles decrease in both cases ( $\mathrm{Gr}<0$ and $\mathrm{Gr}>0)$. The effect of chemical reaction parameter is just like the effect of Hall parameter, which are depicted in Figures 12 and 13. Figure 14 elucidates that the axial velocity profile increases with the increase in 


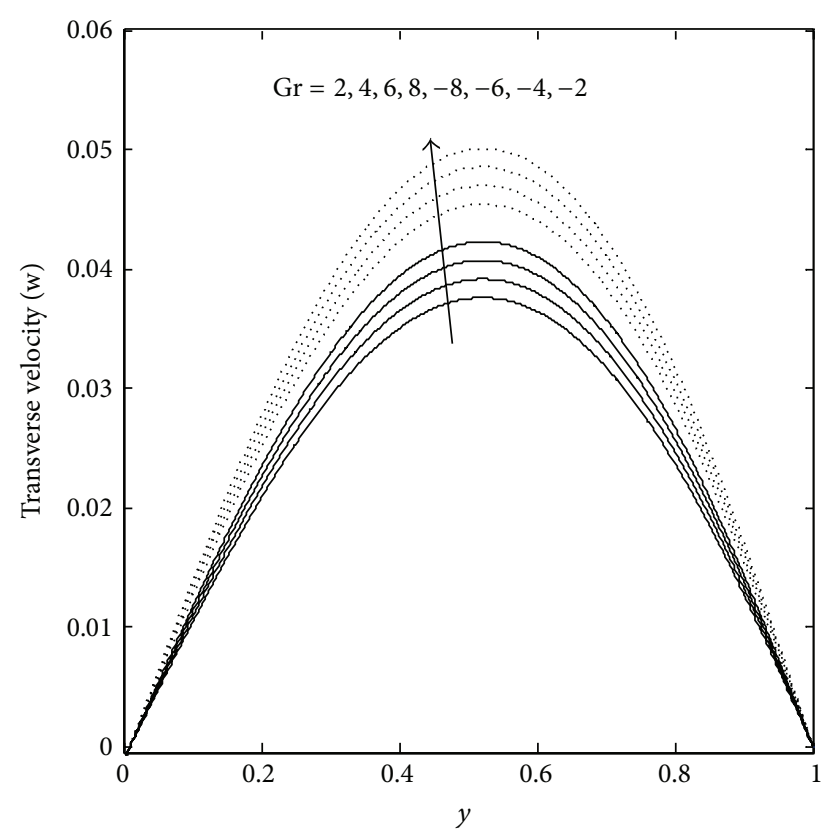

FIGURE 7: Variation of transverse velocity profile for the different values of $\mathrm{Gr}$.

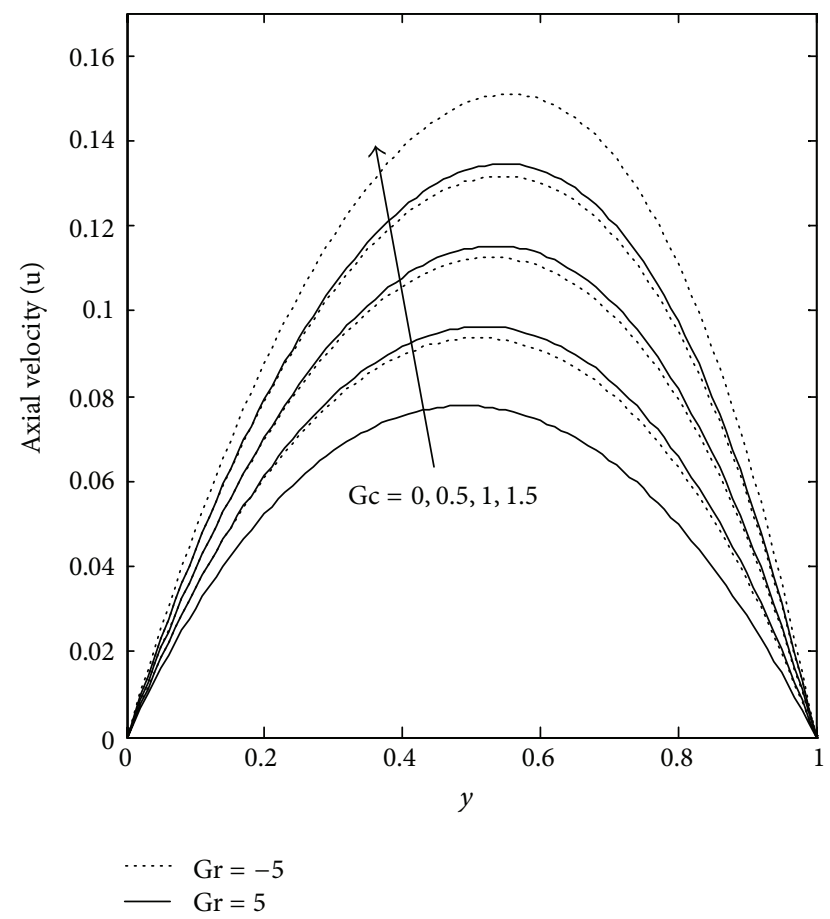

FIGURE 8: Variation of axial velocity profile for the different values of Gc.

the Biot number in the heating case $(\mathrm{Gr}<0)$, and this manner is opposite in cooling case $(\mathrm{Gr}>0)$. Further, the effect of Biot number on transverse velocity profile behaves in similar manner as we noticed in the effect of Biot number on axial velocity field, which is shown in Figure 15. Finally from Figures 2-15, it is interesting to note that both the axial

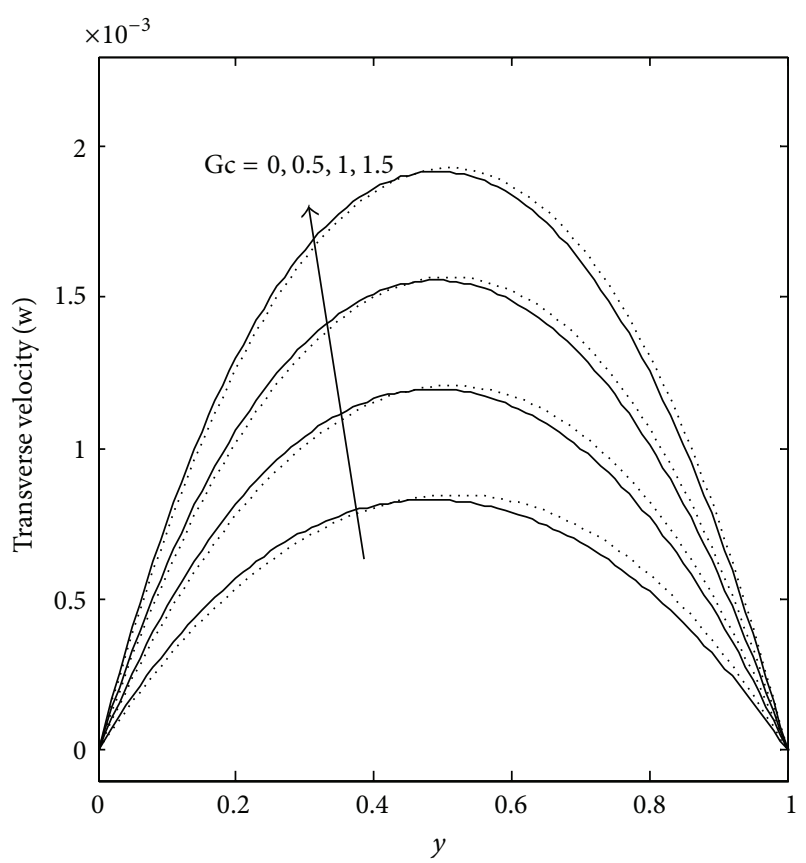

$\cdots \cdot \mathrm{Gr}=-5$

- $\mathrm{Gr}=5$

FIGURE 9: Variation of transverse velocity profile for the different values of $\mathrm{Gc}$.

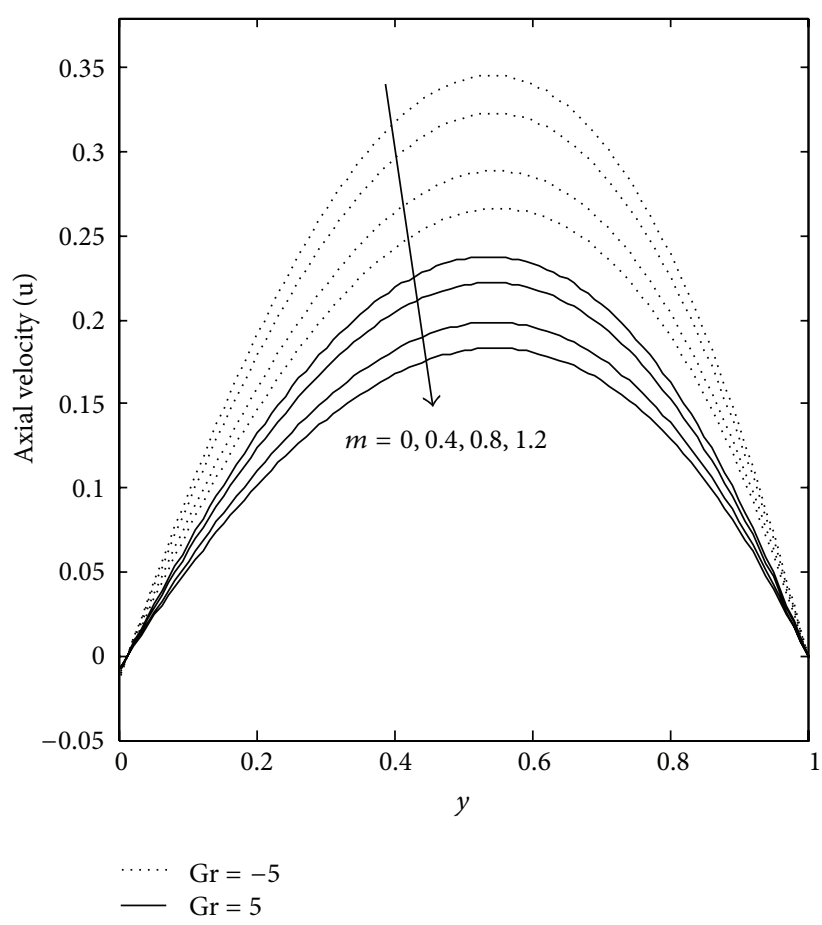

FIGURE 10: Variation of axial velocity profile for the different values of $m$.

and transverse velocity fields are higher in heating case than cooling case. 


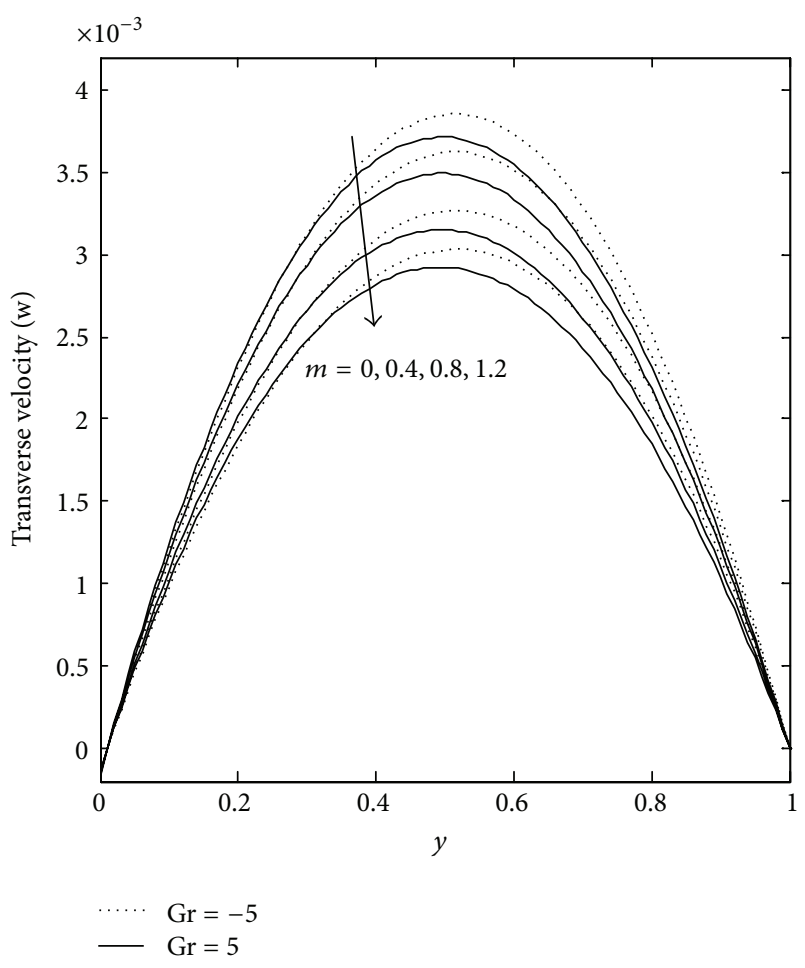

FIGURE 11: Variation of transverse velocity profile for the different values of $m$.

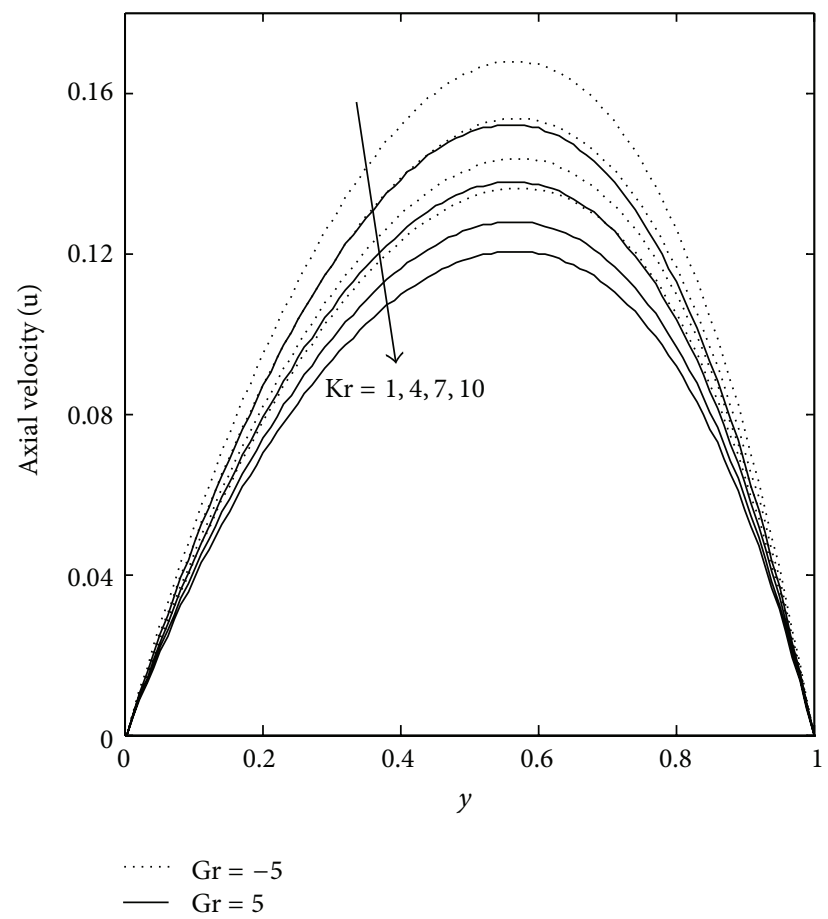

FIGURE 12: Variation of axial velocity profile for the different values of $\mathrm{Kr}$.

Figures 16-19 give the spatial variation of dimensionless temperature distributions for different values $\alpha_{T}, R, \mathrm{Bi}$, and

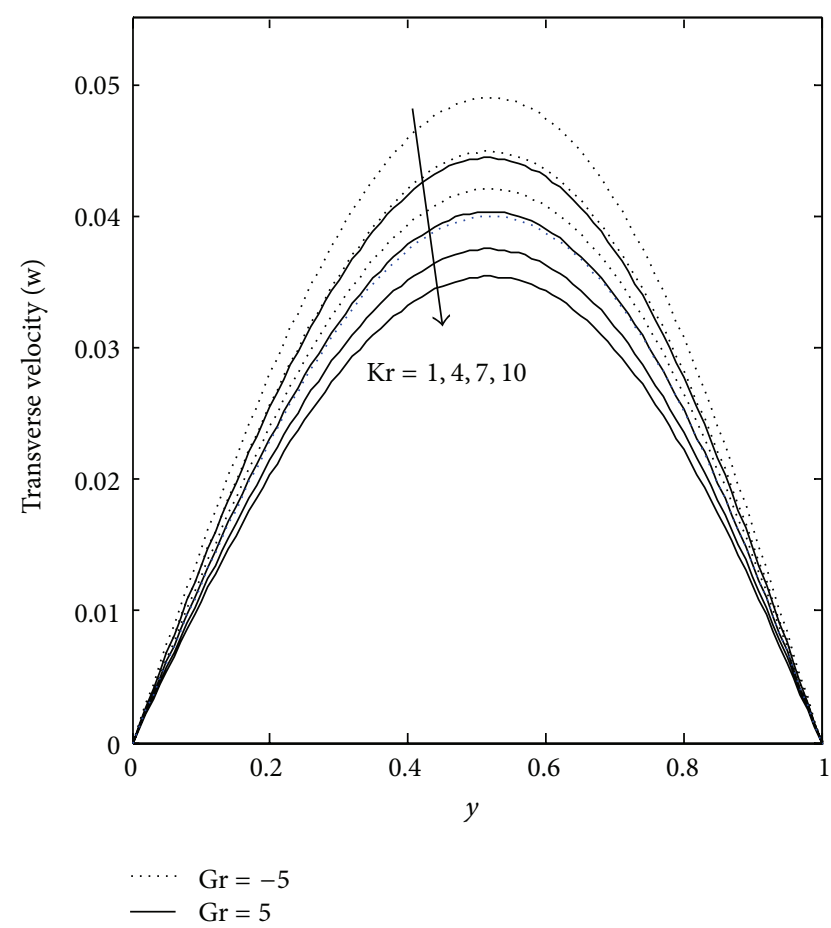

FIGURE 13: Variation of transverse velocity profile for the different values of $\mathrm{Kr}$.

Pr, respectively. Figure 16 disclosed that the temperature profile decreases with increasing values of the heat absorption parameter $\alpha_{T}$ with $\mathrm{Bi}=20$ because the heat absorption parameter has propensity to drop off the thermal buoyancy effect, which decreases the heat transfer. Figure 17 reveals an interesting phenomenon that the temperature profile decreases with the increase in the value of radiation parameter with $\mathrm{Bi}=20$. This result qualitatively agrees with expectations since the effect of radiation is to decrease the rate of energy transport to the fluid, thereby decreasing the temperature of the fluid. The effect of Biot number on temperature profiles is figured in Figure 18. The Biot number is the ratio of the internal thermal resistance of the channel to the boundary layer thermal resistance. When $\mathrm{Bi}=0$ inside of the channel with hot fluid is totally insulated, the internal thermal resistance of the channel is extremely high and no convective heat transfer to the cold fluid on the outside of the channel takes place. Moreover, it is noted that the fluid temperature decreases for the increasing values of Biot number since as $\mathrm{Bi}$ increases, the thermal resistance of the channel decreases and convective heat transfer of the fluid increases. It is expected that as Biot number goes to infinity, the convective boundary conditions will become the prescribed wall temperature case [18].

Figure 19 has been plotted to depict the variation of temperature profiles against $y$ for different values of Prandtl number by fixing other physical parameters $(\mathrm{Bi}=2)$. Four different values of Prandtl number, namely, $\mathrm{Pr}=0.71,1,3$, and 7 corresponding to air, electrolyte, Freon, and water, respectively, are chosen. From this graph we have observed that temperature profile decreases with the increase in the Prandtl 


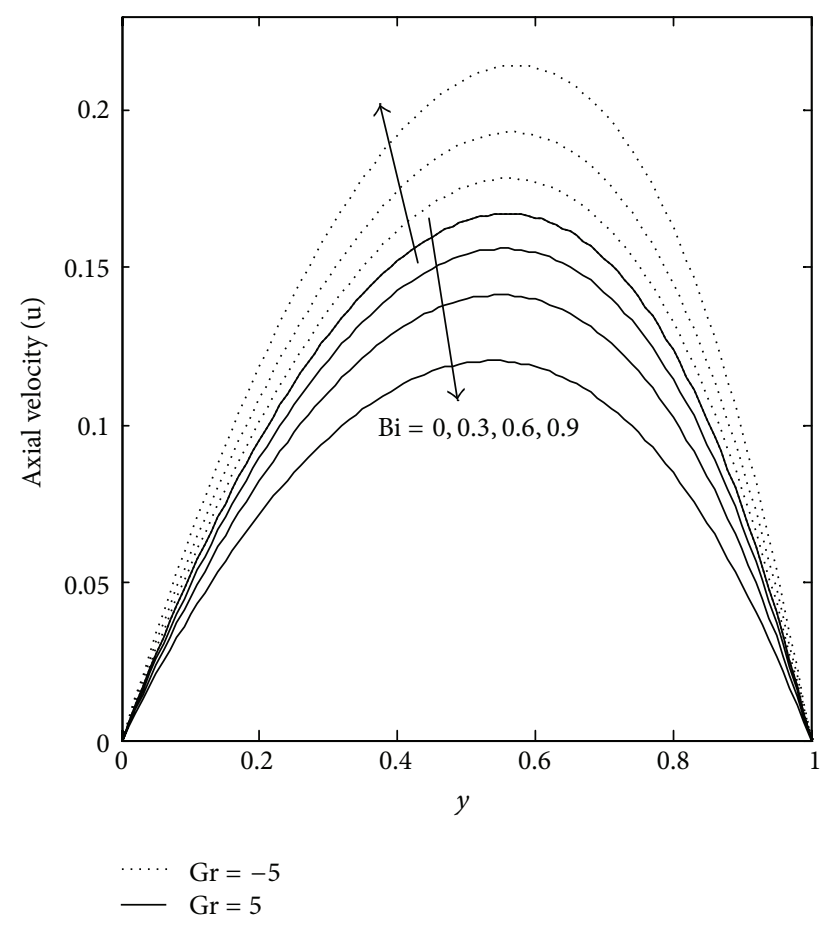

FIGURE 14: Variation of axial velocity profile for the different values of $\mathrm{Bi}$.

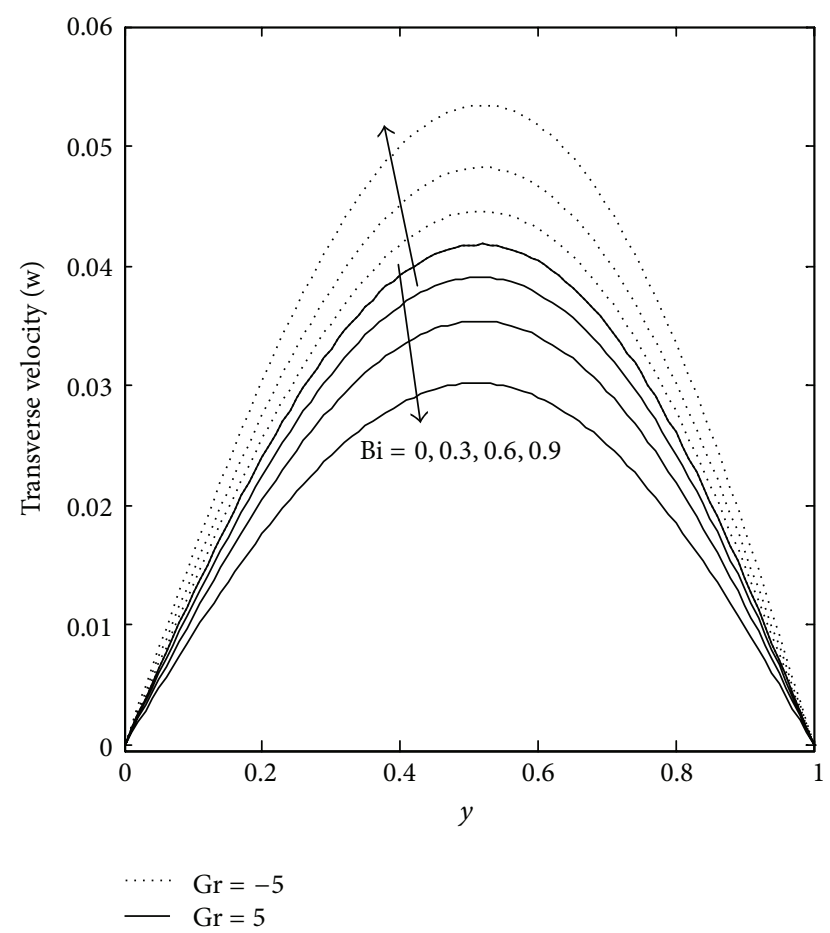

FIGURE 15: Variation of transverse velocity profile for the different values of $\mathrm{Bi}$.

number. This is because fluids with large Pr have low thermal diffusivity which cause low heat penetration resulting in reducing the temperature distributions.

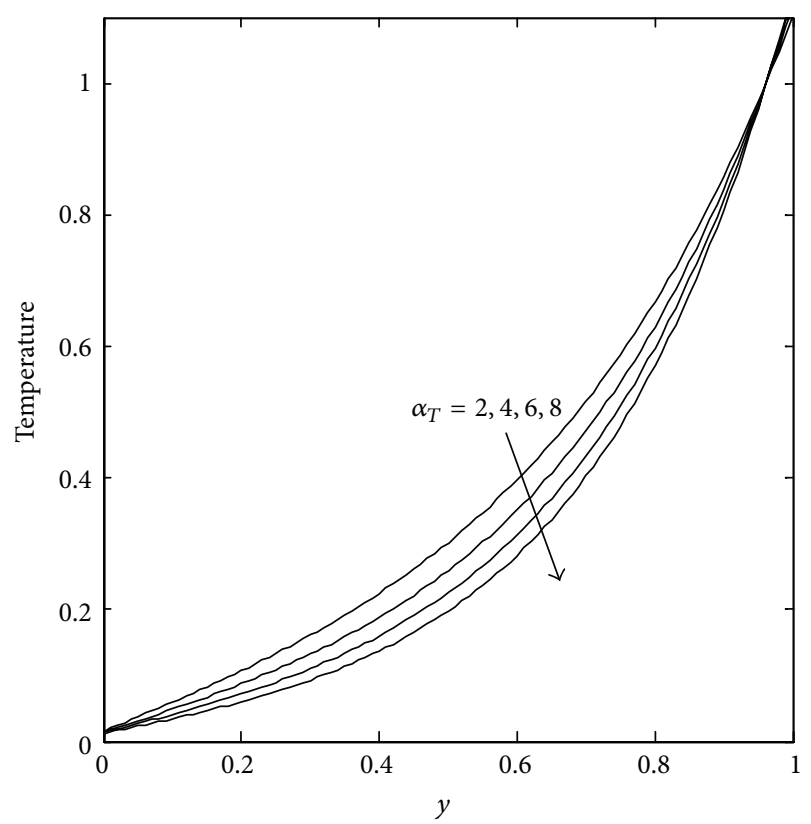

FIGURE 16: Variation of temperature profile for the different values of $\alpha_{T}$.

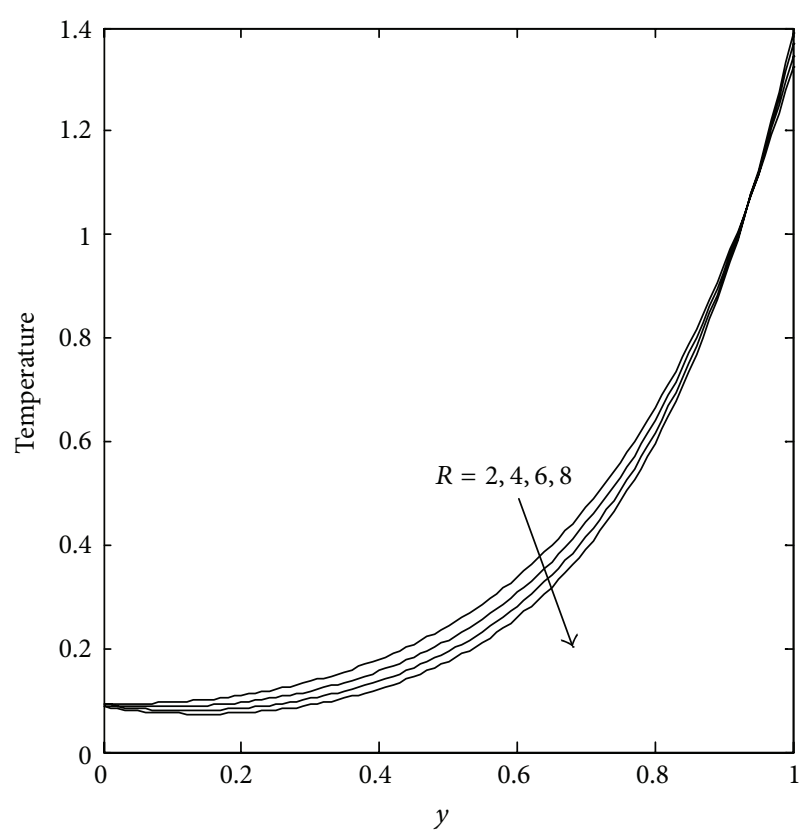

FIGURE 17: Variation of temperature profile for the different values of $R$.

The effect of the chemical reaction parameter on the species concentration profile is shown in Figure 20. It is clearly observed that the concentration profile decreases for increasing values of chemical reaction parameter. This is due to the fact that destructive chemical reaction reduces the solutal boundary layer thickness and decreases the mass transfer. Figure 21 displays concentration profiles versus $y$ for various gases like hydrogen $(S c=0.22)$, helium $(S c=0.30)$, water vapour $(\mathrm{Sc}=0.60)$, and ammonia $(\mathrm{Sc}=0.78)$. It 


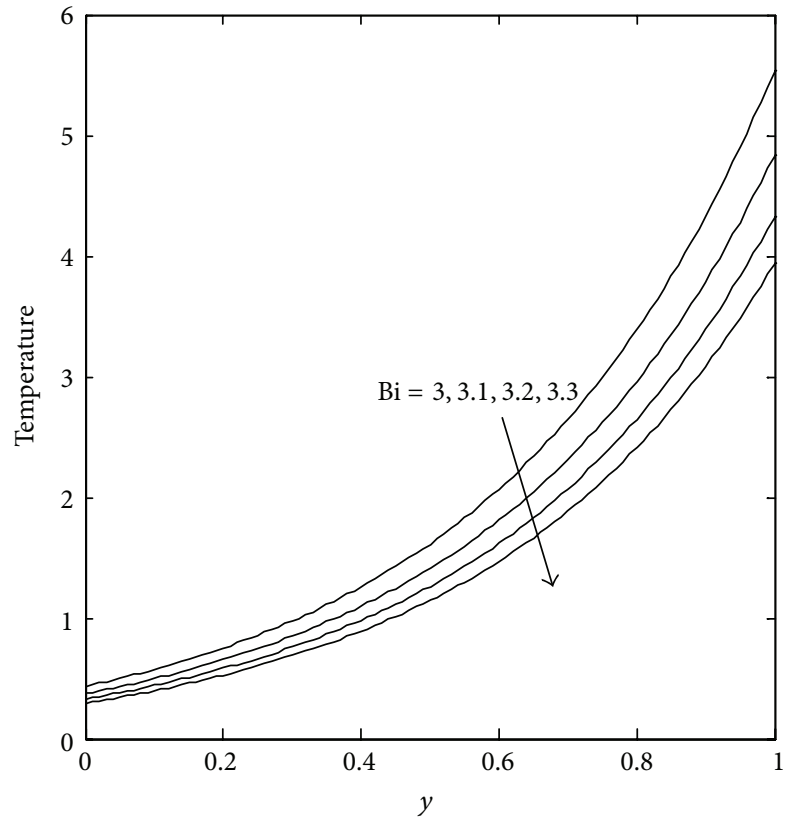

FIGURE 18: Variation of temperature profile for the different values of $\mathrm{Bi}$.

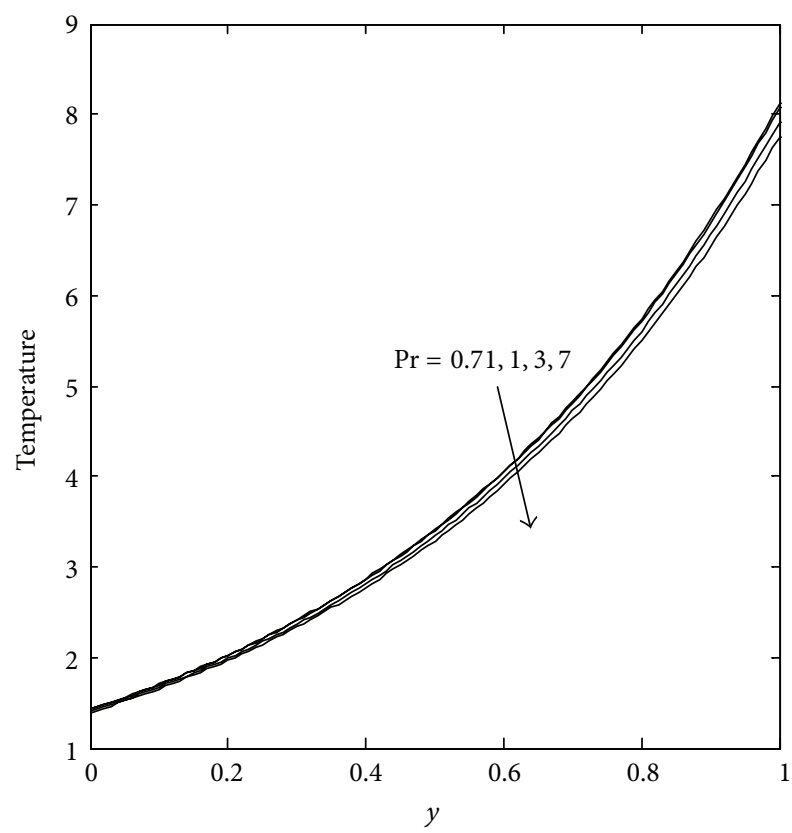

FIGURE 19: Variation of temperature profile for the different values of Pr.

is elucidated that an increase in Schmidt number increases the conduction, which decreases the mass transfer because the increase of Sc means a decrease of molecular diffusivity that results in decrease of concentration profile. Hence the concentration of species is higher for smaller value of Sc and vice versa.

Figures 22 and 23 are plotted to exemplify the effect of magnetic parameter and Hall parameter on skin friction

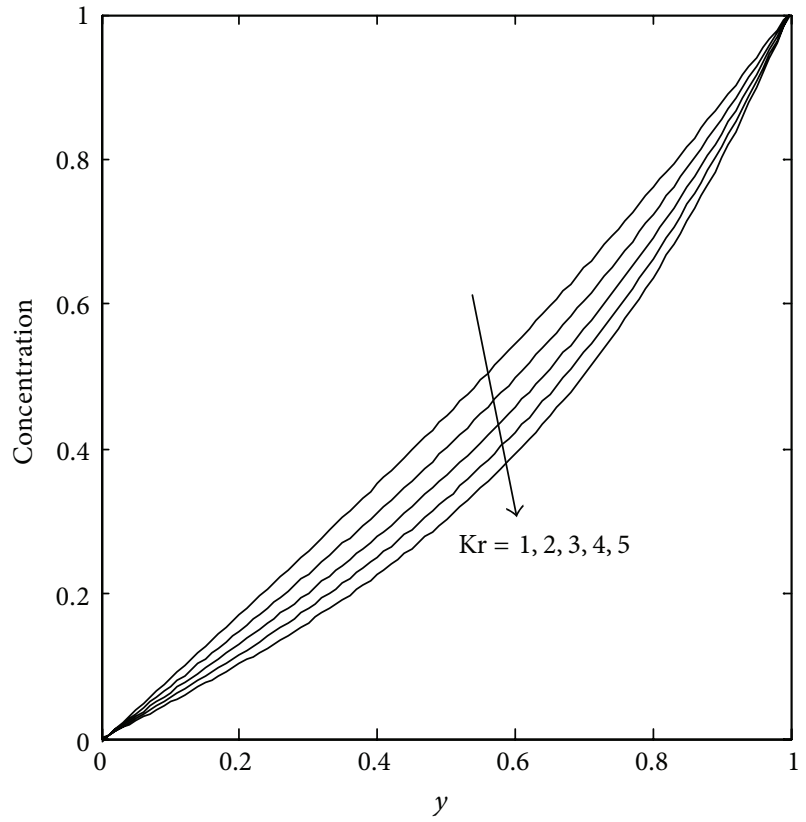

FIGURE 20: Variation of concentration profile for the different values of $\mathrm{Kr}$.

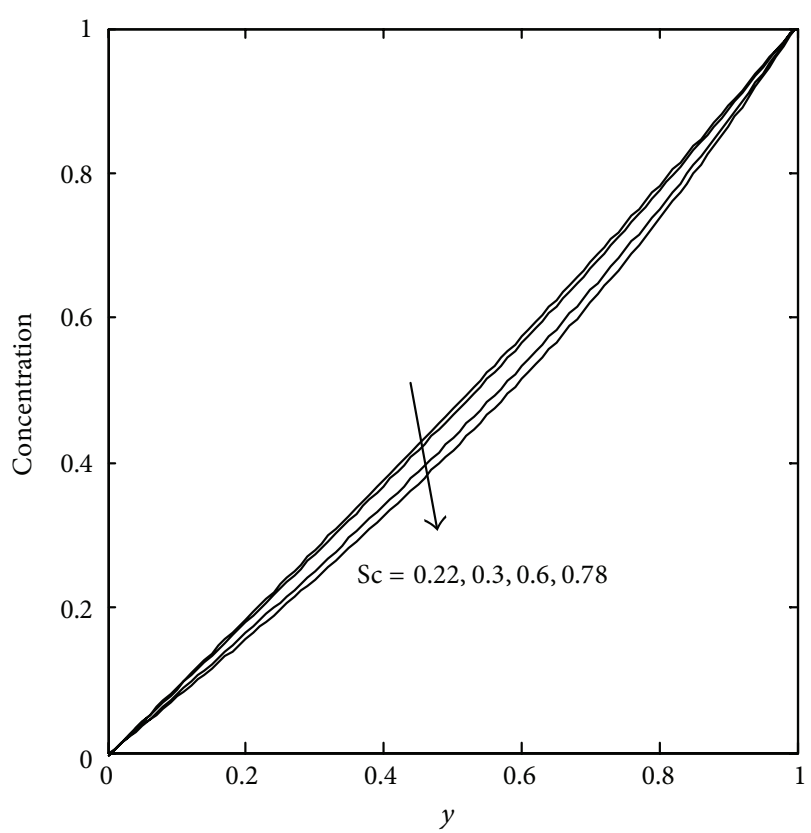

FIGURE 21: Variation of concentration profile for the different values of Sc.

components $\left(\tau_{1}, \tau_{2}\right)$ at the wavy wall $(y=h)$ in both cases $(\mathrm{Gr}<0$ and $\mathrm{Gr}>0)$, respectively. Figure 22 illustrates that, the axial skin friction $\left(\tau_{1}\right)$ coefficient remarkably decreases for increasing values of magnetic parameter in both cases, while this trend is opposite for transverse skin friction $\left(\tau_{2}\right)$ coefficient in both cases. The axial skin friction $\left(\tau_{1}\right)$ profile enhances increasing values of Hall parameter in both the 
TABLE 1: Effects of $E, t, \mathrm{Bi}, \mathrm{Kr}$, and Gc on skin friction distributions at the wavy wall $y=h$ for both cooling $(\mathrm{Gr}>0)$ and heating $(\mathrm{Gr}<0)$ cases.

\begin{tabular}{|c|c|c|c|c|c|c|c|c|}
\hline$E$ & $t$ & $\mathrm{Bi}$ & $\mathrm{Kr}$ & Gc & $\begin{array}{c}\tau_{1} \\
\mathrm{Gr}<0\end{array}$ & $\begin{array}{c}\tau_{2} \\
\mathrm{Gr}<0\end{array}$ & $\begin{array}{c}\tau_{1} \\
\mathrm{Gr}>0\end{array}$ & $\begin{array}{c}\tau_{2} \\
\mathrm{Gr}>0\end{array}$ \\
\hline 2.0 & 0.1 & 0.1 & 2.0 & 2.0 & 0.5139 & 0.1411 & 0.4591 & 0.1244 \\
\hline 4.0 & & & & & 0.4200 & 0.2202 & 0.3767 & 0.1941 \\
\hline 6.0 & & & & & 0.3289 & 0.2437 & 0.2967 & 0.2153 \\
\hline \multirow[t]{3}{*}{1.0} & 0.0 & 0.1 & 2.0 & 2.0 & 0.5690 & 0.0780 & 0.4471 & 0.0596 \\
\hline & 2.0 & & & & 0.5699 & 0.0787 & 0.4476 & 0.0601 \\
\hline & 4.0 & & & & 0.5735 & 0.0818 & 0.4515 & 0.0631 \\
\hline \multirow[t]{3}{*}{1.0} & 0.1 & 0.1 & 2.0 & 2.0 & 0.5470 & 0.0784 & 0.4882 & 0.0694 \\
\hline & & 0.3 & & & 0.6121 & 0.0885 & 0.4231 & 0.0593 \\
\hline & & 0.5 & & & 0.6888 & 0.1007 & 0.3464 & 0.0472 \\
\hline \multirow[t]{3}{*}{1.0} & 0.1 & 0.1 & 1.0 & 2.0 & 0.5558 & 0.0776 & 0.4970 & 0.0687 \\
\hline & & & 5.0 & & 0.5200 & 0.0766 & 0.4612 & 0.0676 \\
\hline & & & 9.0 & & 0.5011 & 0.0697 & 0.4423 & 0.0607 \\
\hline \multirow[t]{3}{*}{1.0} & 0.1 & 0.1 & 2.0 & 1.0 & 0.5731 & 0.0815 & 0.4510 & 0.0628 \\
\hline & & & & 2.0 & 0.6792 & 0.1038 & 0.5571 & 0.0851 \\
\hline & & & & 3.0 & 0.7853 & 0.1262 & 0.6633 & 0.1075 \\
\hline
\end{tabular}

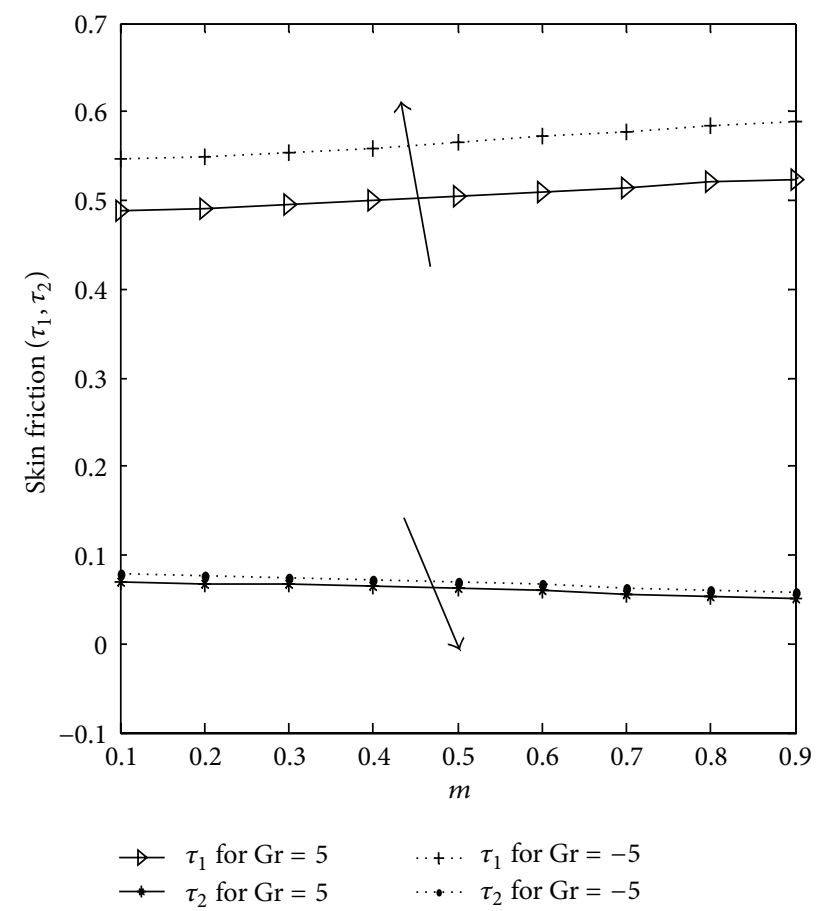

FIGURE 22: Variation of skin friction profile for the different values of $M^{2}$.

cases, but this manner is reverse for transverse skin friction coefficient, which is depicted from Figure 23.

Table 1 illustrates the variation of skin friction components at the wavy wall $(y=h)$ for different values of $E, t$, $\mathrm{Bi}, \mathrm{Kr}$, and Gc. Further Tables 2 and 3 illustrate the variation of the Nusselt number and Sherwood number at the wavy wall $y=h$, for different values of $\operatorname{Pr}, t$, and $R$ and $\mathrm{Kr}, t$, and $\mathrm{Sc}$, respectively. Throughout the computations the values of

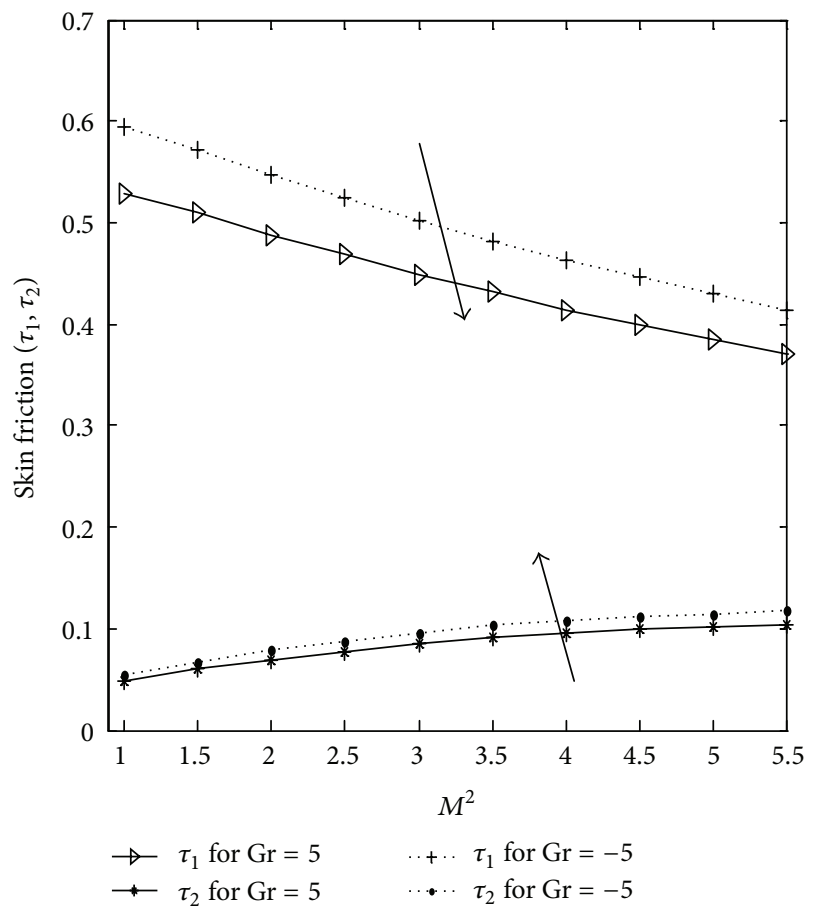

FIGURE 23: Variation of skin friction profile for the different values of $m$.

physical parameters $\mathrm{Kr}=2, t=0.1, E=1, k_{0}=2, \operatorname{Pr}=3$, $\mathrm{Gc}=2, \mathrm{Sc}=0.78, \alpha_{T}=1, \mathrm{Bi}=0.1, R=4, M^{2}=2$, $A=B=1, n=0.1, \epsilon=0.02, m=0.1$, and $\lambda=2 \pi$ are used, unless otherwise stated.

\section{Conclusions}

The present investigation dealt with the combined effect of thermal radiation and Hall current on unsteady MHD 
TABle 2: Effects of Pr, $t$, and $R$ on Nusselt number distributions at the wavy wall $y=h$.

\begin{tabular}{lcc}
\hline Parameter & Values & $\mathrm{Nu}$ \\
\hline \multirow{3}{*}{ Pr } & 0.0 & 2.6676 \\
& 0.72 & 2.5993 \\
& 3.0 & 2.5613 \\
\hline \multirow{2}{*}{$t$} & 0.5 & 2.6033 \\
& 1.5 & 2.5767 \\
& 2.5 & 2.5647 \\
\hline \multirow{2}{*}{$R$} & 0.0 & 1.1447 \\
& 1.0 & 1.4854 \\
& 2.0 & 2.5993 \\
\hline
\end{tabular}

TABLE 3: Effects of Kr, $t$, and Sc on Sherwood number distributions at the wavy wall $y=h$.

\begin{tabular}{lcc}
\hline Parameter & Values & Sh \\
\hline \multirow{3}{*}{$\mathrm{Kr}$} & 1.0 & 1.8162 \\
& 3.0 & 1.4499 \\
& 5.0 & 1.1138 \\
\hline \multirow{2}{*}{$t$} & 0.0 & 0.6650 \\
& 1.0 & 0.7933 \\
& 2.0 & 0.8405 \\
Sc & 0.22 & 0.9990 \\
& 0.30 & 0.9599 \\
& 0.78 & 0.7409 \\
\hline
\end{tabular}

visco-elastic fluid flow in an irregular channel subject to convective boundary condition. The study is also concerned with the free convective flow and mass transfer with heat absorption and chemical reaction effects. The perturbation method is used to solve the problem, and the results are evaluated numerically and displayed graphically using MAT$\mathrm{LAB}$ package. In the light of the present investigation, the following conclusions can be summarized.

(i) The velocity profiles are parabolic in nature, and the velocity field is higher in heating $(\mathrm{Gr}<0)$ case than that in cooling $(\mathrm{Gr}>0)$ case.

(ii) The Hall effect plays an important role in controlling momentum of the fluid.

(iii) The strength of the applied magnetic field should be as low as possible to realize.

(iv) The temperature profile decreases for increasing values of heat absorption parameter.

(v) The effect of increasing values of Prandtl number decreases temperature distributions.

(vi) An increase in the dimensionless time enhances the skin friction profile at the wavy wall, and this manner is opposite for increasing values of chemical reaction parameter.

(vii) The rate of heat transfer decreases for increasing values of heat absorption parameter at the wavy wall. (viii) Combined effect of chemical reaction parameter and Schmidt number reduces the rate of mass transfer at the wavy wall, but this trend is reverse for the increasing in dimensionless time.

Thus the present study will serve as a good scientific tool for understanding more complex flow problems concerning the various physical parameters.

\section{Acknowledgments}

The authors wish to express their deep sense of gratitude to the editor and referee for their encouraging suggestions that significantly improved the paper.

\section{References}

[1] D. W. Beard and K. Walters, "Elastico-viscous boundary layer flows I. Two dimensional flow near a stagnation point," Mathematical Proceedings of the Cambridge Philosophical Society, vol. 60, pp. 667-674, 1964.

[2] K. R. Rajagopal and T. Y. Na, "On Stokes' problem for a nonNewtonian fluid," Acta Mechanica, vol. 48, no. 3-4, pp. 233-239, 1983.

[3] T. Sarpkaya, "Flow of non-Newtonian fluids in a magnetic field," AIChE Journal, vol. 7, pp. 324-328, 1961.

[4] C. C. Chang and T. S. Lundgren, "Duct flow in magnetohydrodynamics," Zeitschrift für angewandte Mathematik und Physik, vol. 12, no. 2, pp. 100-114, 1961.

[5] H. A. Attia and K. M. Ewis, "Unsteady MHD couette flow with heat transfer of a viscoelastic fluid under exponential decaying pressure gradient," Tamkang Journal of Science and Engineering, vol. 13, no. 4, pp. 359-364, 2010.

[6] M. E. Sayed-Ahmed and H. A. Attia, "MHD flow and heat transfer in a rectangular duct with temperature dependent viscosity and Hall effect," International Communications in Heat and Mass Transfer, vol. 27, no. 8, pp. 1177-1187, 2000.

[7] H. A. Attia and M. A. M. Abdeen, "Unsteady hartmann flow with heat transfer of a viscoelastic fluid under exponential decaying pressure gradient," Engineering MECHANICS, vol. 19, no. 5, pp. 37-44, 2012.

[8] H. A. Attia, "Hall effect on couette flow with heat transfer of a dusty conducting fluid between parallel porous plates under exponential decaying pressure gradient," Journal of Mechanical Science and Technology, vol. 20, no. 4, pp. 569-579, 2006.

[9] T. Hayat and O. U. Mehmood, "Slip effects on MHD flow of third order fluid in a planar channel," Communications in Nonlinear Science and Numerical Simulation, vol. 16, no. 3, pp. 1363-1377, 2011.

[10] Kh. S. Mekheimer and M. A. El Kot, "Influence of magnetic field and Hall currents on blood flow through a stenotic artery," Applied Mathematics and Mechanics, vol. 29, no. 8, pp. 10931104, 2008.

[11] D. Pal and B. Talukdar, "Perturbation analysis of unsteady magnetohydrodynamic convective heat and mass transfer in a boundary layer slip flow past a vertical permeable plate with thermal radiation and chemical reaction," Communications in Nonlinear Science and Numerical Simulation, vol. 15, no. 7, pp. 1813-1830, 2010.

[12] A. J. Chamkha, "MHD flow of a uniformly streched vertical permeable surface in the presence of heat generation/absorption 
and a chemical reaction," International Communications in Heat and Mass Transfer, vol. 30, no. 3, pp. 413-422, 2003.

[13] I. J. Uwanta and E. Omokhuale, "Viscoelastic fluid flow in a fixed plane with heat and mass transfer," Research Journal of Mathematics and Statistics, vol. 4, no. 3, pp. 63-69, 2012.

[14] S. S. Saxena and G. K. Dubey, "MHD free convection heat and mass transfer flow of viscoelastic fluid embedded in a porous medium of variable permeability with radiation effect and heat source in slip flow regime," Advances in Applied Science Research, vol. 2, no. 5, pp. 115-129, 2011.

[15] M. Massoudi and A. K. Uguz, "Chemically-reacting fluids with variable transport properties," Applied Mathematics and Computation, vol. 219, pp. 1761-1775, 2012.

[16] R. C. Bataller, "Radiation effects for the Blasius and Sakiadis flows with a convective surface boundary condition," Applied Mathematics and Computation, vol. 206, no. 2, pp. 832-840, 2008.

[17] O. D. Makinde and T. Chinyoka, "Numerical study of unsteady hydromagnetic Generalized Couette flow of a reactive thirdgrade fluid with asymmetric convective cooling," Computers and Mathematics with Applications, vol. 61, no. 4, pp. 1167-1179, 2011.

[18] S. Yao, T. Fang, and Y. Zhong, "Heat transfer of a generalized stretching/shrinking wall problem with convective boundary conditions," Communications in Nonlinear Science and Numerical Simulation, vol. 16, no. 2, pp. 752-760, 2011.

[19] O. D. Makinde and A. Aziz, "MHD mixed convection from a vertical plate embedded in a porous medium with a convective boundary condition," International Journal of Thermal Sciences, vol. 49, no. 9, pp. 1813-1820, 2010.

[20] G. W. Sutton and A. Sherman, Engineering Magnetohydrodynamics, McGraw-Hill, New York, NY, USA, 1965.

[21] T. Hayat and M. Nawaz, "Soret and Dufour effects on the mixed convection flow of a second grade fluid subject to Hall and ionslip currents," International Journal for Numerical Methods in Fluids, vol. 67, no. 9, pp. 1073-1099, 2011.

[22] U. N. Das, "Free convective MHD flow and heat transfer in a viscous incompressible fluid confined between a long vertical wavy wall and a parallel flat wall," Indian Journal of Pure and Applied Mathematics, vol. 23, pp. 295-304, 1992.

[23] Y. Abd elmaboud and Kh. S. Mekheimer, "Unsteady pulsatile flow through a vertical constricted annulus with heat transfer," Zeitschrift für Naturforschung A, vol. 67, pp. 185-194, 2012.

[24] B. Ničeno and E. Nobile, "Numerical analysis of fluid flow and heat transfer in periodic wavy channels," International Journal of Heat and Fluid Flow, vol. 22, no. 2, pp. 156-167, 2001.

[25] Kh. S. Mekheimer, S. Z. A. Husseny, and Y. Abd Elmaboud, "Effects of heat transfer and space porosity on peristaltic flow in a vertical asymmetric channel," Numerical Methods for Partial Differential Equations, vol. 26, no. 4, pp. 747-770, 2010.

[26] R. Sivaraj and B. Rushi Kumar, "Unsteady MHD dusty viscoelastic fluid Couette flow in an irregular channel with varying mass diffusion," International Journal of Heat and Mass Transfer, vol. 55, no. 11-12, pp. 3076-3089, 2012.

[27] R. Taneja and N. C. Jain, "MHD flow with slip effects and temperature-dependent heat source in a viscous incompressible fluid confined between a long vertical wavy wall and a parallel flat wall," Defence Science Journal, vol. 54, no. 1, pp. 21-29, 2004.

[28] K. Walters, On Second-Order Effect in Elasticity,Plasticity and Fluid Mechanics, IUTAM Int. Symp., Pergamon Press, New York, NY, USA, 1964.
[29] A. C. Cogley, S. E. Giles, and W. G. Vincent, "Differential approximation to radiative heat transfer in a nongrey gas near equilibrium," AIAA Journal, vol. 6, no. 3, pp. 551-553, 1968. 

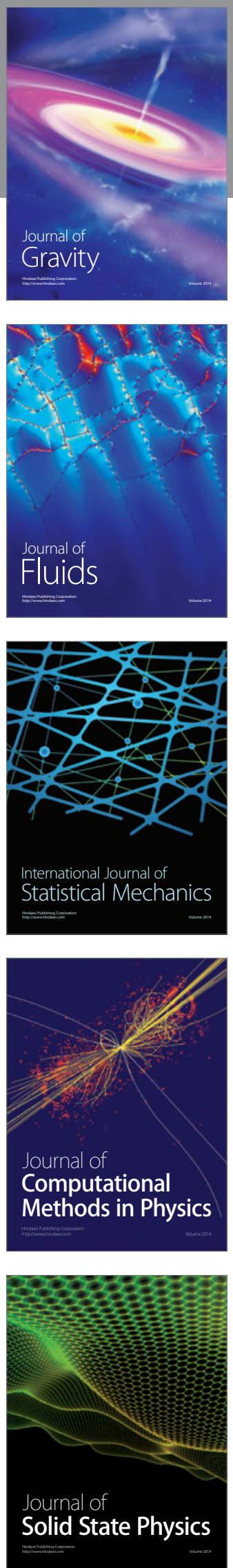

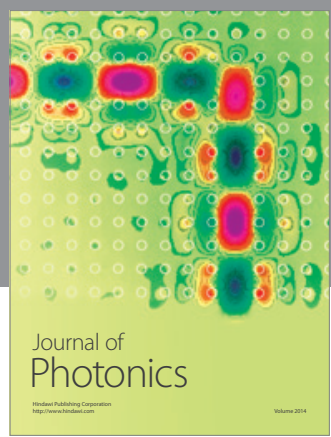

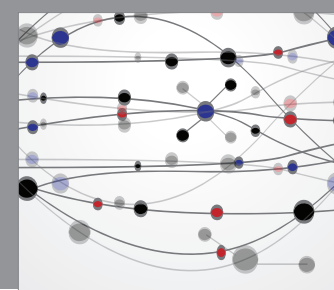

The Scientific World Journal

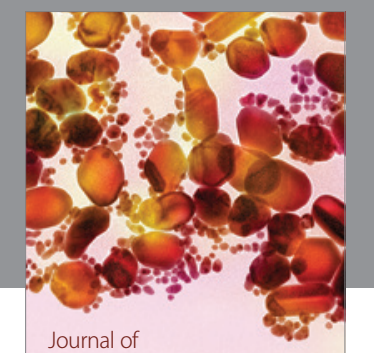

Soft Matter
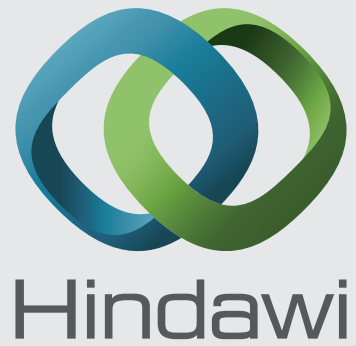

Submit your manuscripts at

http://www.hindawi.com
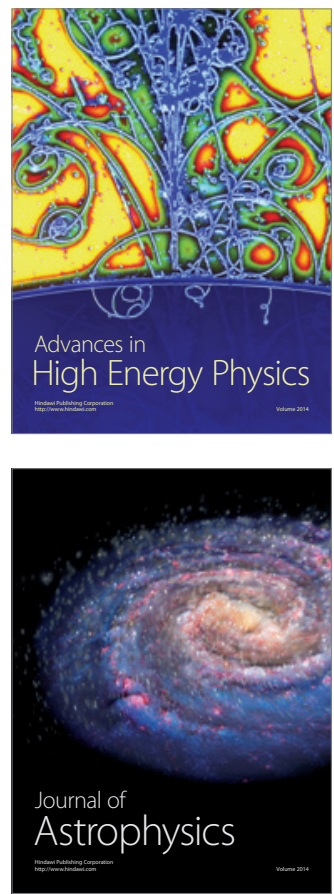
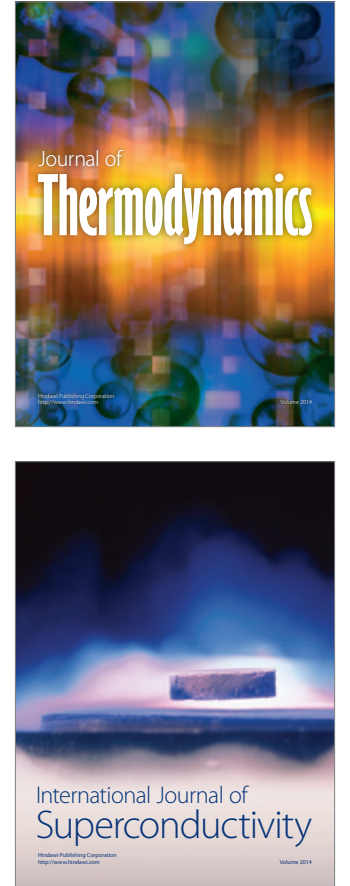
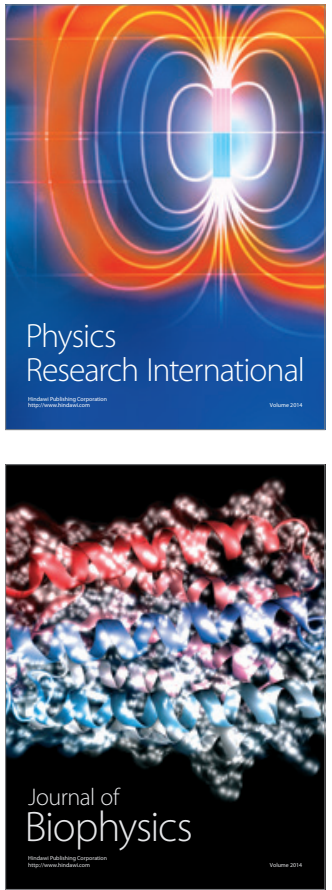
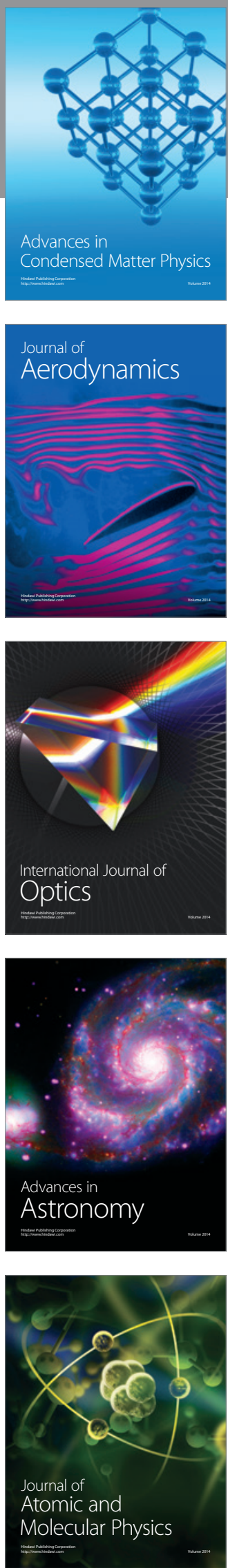